\title{
Low-Order Stochastic Mode Reduction for a Prototype Atmospheric GCM
}

\author{
Christian Franzke And Andrew J. Majda \\ Courant Institute of Mathematical Sciences, Center for Atmosphere-Ocean Science, New York University, New York, New York
}

(Manuscript received 10 October 2004, in final form 3 March 2005)

\begin{abstract}
This study applies a systematic strategy for stochastic modeling of atmospheric low-frequency variability to a three-layer quasigeostrophic model. This model climate has reasonable approximations of the North Atlantic Oscillation (NAO) and Pacific-North America (PNA) patterns. The systematic strategy consists first of the identification of slowly evolving climate modes and faster evolving nonclimate modes by use of an empirical orthogonal function (EOF) decomposition in the total energy metric. The low-order stochastic climate model predicts the evolution of these climate modes a priori without any regression fitting of the resolved modes. The systematic stochastic mode reduction strategy determines all correction terms and noises with minimal regression fitting of the variances and correlation times of the unresolved modes. These correction terms and noises account for the neglected interactions between the resolved climate modes and the unresolved nonclimate modes. Low-order stochastic models with 10 or less resolved modes capture the statistics of the original model very well, including the variances and temporal correlations with high pattern correlations of the transient eddy fluxes. A budget analysis establishes that the low-order stochastic models are highly nonlinear with significant contributions from both additive and multiplicative noise. This is in contrast to previous stochastic modeling studies. These studies a priori assume a linear model with additive noise and regression fit the resolved modes. The multiplicative noise comes from the advection of the resolved modes by the unresolved modes. The most straightforward low-order stochastic climate models experience climate drift that stems from the bare truncation dynamics. Even though the geographic correlation of the transient eddy fluxes is high, they are underestimated by a factor of about 2 in the a priori procedure and thus cannot completely overcome the large climate drift in the bare truncation. Also, variants of the reduced stochastic modeling procedure that experience no climate drift with good predictions of both the variances and time correlations are discussed. These reduced models without climate drift are developed by slowing down the dynamics of the bare truncation compared with the interactions with the unresolved modes and yield a minimal two-parameter regression fitting strategy for the climate modes. This study points to the need for better optimal basis functions that optimally capture the essential slow dynamics of the system to obtain further improvements for the reduced stochastic modeling procedure.
\end{abstract}

\section{Introduction}

A remarkable fact of Northern Hemisphere lowfrequency variability is that it can be efficiently described by only a few teleconnection patterns that explain most of the total variance (e.g., Wallace and Gutzler 1981). These few teleconnection patterns not only exert a strong influence on regional climate and weather, they are also related to climate change (Hurrell 1995; Thompson and Wallace 2001). These properties of teleconnection patterns make them an attractive

Corresponding author address: Dr. Christian Franzke, Courant Institute of Mathematical Sciences, New York University, 251 Mercer Street, New York, NY 10012.

E-mail: franzke@cims.nyu.edu choice as basis functions for climate models with a highly reduced number of degrees of freedom.

The development of such reduced climate models involves the solution of two major issues: 1) how to properly account for the unresolved modes, also known as the closure problem; and 2) how to define a small set of basis functions that optimally represent the dynamics of the major teleconnection patterns. This study primarily addresses issue 1 and presents a rigorous strategy of how to systematically account for the unresolved degrees of freedom.

The simplest approach to derive highly truncated models of teleconnection patterns is to empirically fit simple stochastic models (e.g., autoregressive models and fractionally differenced models) to individual scalar teleconnection indices (Feldstein 2000, 2002; 
Stephenson et al. 2000; Percival et al. 2001). Statistical tests usually cannot distinguish if short- or longmemory models provide the better fit.

A more complex approach, which also tries to capture deterministic interactions between different teleconnection patterns, is to linearize the equations of motion around a climatological mean state. Such models can be determined empirically from data or by using the linearized equations of motion. These models can either be forced by a random forcing (Branstator 1990; Branstator and Frederiksen 2003; Farrell and Ioannou 1993; Newman et al. 1997; Whitaker and Sardeshmukh 1998; Zhang and Held 1999; Winkler et al. 2001) or by an external forcing representing tropical heating (Branstator and Haupt 1998). To ensure stability of these linear models damping is added according to various ad hoc principles. There is a recent survey of such modeling strategies (Delsole 2004).

A more powerful method is to empirically fit nonlinear stochastic models with possibly multiplicative (state dependent) noise by using the Fokker-Planck equation (Gardiner 1985; Sura and Barsugli 2002; Sura 2003; Berner 2005). To reliably estimate the drift and diffusion coefficients in the Fokker-Planck equation is a subtle inverse problem that requires very long time series and is further complicated by the need to retain the leading order eigenvalue structure of the FokkerPlanck operator in order to keep the autocorrelation time scales of the original model (D. Crommelin and E. Vanden-Eijnden 2005, personal communication). Kravtsov et al. (2005) and Kondrashov et al. (2006) apply an interesting quadratically nonlinear multilevel regression strategy to fit suitable climate mode dynamics for a three-level quasigeostrophic model (Marshall and Molteni 1993; the model is used in this study). For 15 climate modes, they regression fit 3165 parameters to achieve very good results.

All the work presented above derives reduced models by regression fitting of the resolved modes. Another approach is to take advantage of the basis function property of teleconnection patterns. Schubert (1985), Selten (1993, 1995, 1997), Achatz and Branstator (1999), D'Andrea and Vautard (2001), and Achatz and Opsteegh (2003a, 2003b) developed low-order models with EOFs as basis functions. Truncated EOF models experience climate drift because of the neglected interactions with the unresolved modes. Selten (1995) and Achatz and Branstator (1999) parameterize these neglected interactions by a linear damping, whose strength is determined empirically. A possibly more powerful tool to represent the dynamics of a system are principal interaction patterns (PIP; Hasselmann 1988;
Kwasniok 1996, 2004). The calculation of PIPs takes into account the dynamics of the model for which one tries to find an optimal basis and also often involves ad hoc closure through linear damping and an ansatz for nonlinear interactions. Crommelin and Majda (2004) compare different optimal bases. They find that models based on PIPs are superior to models based on EOFs and optimal persistence patterns (Delsole 2001). On the other hand, they also point out that the determination of PIPs can show sensitivities regarding the calculation procedure, at least for some low-order atmospheric dynamical systems with regime transitions. This feature can make PIPs possibly a less attractive basis.

Majda et al. (1999, 2001, 2002, 2003, 2005, manuscript submitted to Nonlinearity, hereafter MTV99, MTV01, etc.) provide a systematic framework for how to account for the effect of the unresolved degrees of freedom on the resolved modes in combination with using the dominant teleconnection patterns as basis functions. In contrast to the empirical fitting procedures applied in the studies discussed above the stochastic mode reduction strategy put forward in MTV predicts all deterministic and stochastic correction terms with only minimal regression fitting of only the unresolved modes. In general only an estimate for the variance and eddy turnover time for each unresolved mode is needed. Such properties for variance and eddy turnover time can often be determined by a single parameter in various turbulent cascade scaling regimes (MTV02; MTV05; Majda and Timofeyev 2004). It has been applied and tested on a wide variety of simplified models and examples. The idealized models, where the procedure has been tested, include those with trivial climates (MTV02), periodic orbits or multiple equilibria (MTV03), and heteroclinic chaotic orbits coupled to a deterministic bath of modes satisfying the truncated Burgers equation (Majda and Timofeyev 2000, 2004); the MTV procedure has been validated in these examples even when there is little separation of time scales between resolved and unresolved modes. An especially stringent recent test is the application of this procedure to the first few large-scale modes of the truncated Burgers equations itself (MTV05). To investigate the influence of topographic stress on the angular momentum budget, they applied this strategy to idealized flow over topography and derived a nonlinear reduced stochastic model with multiplicative noises (MTV03). This stochastic model turns out to be superior compared to a standard linear model with damping and white noise forcing. Also the explicit assumptions of varying the correlation times between the resolved 
modes and the unresolved modes in the MTV procedure for the climate model were checked explicitly by varying the topographic height (MTV03).

Franzke et al. (2005) put the above systematic stochastic mode reduction strategy in a form that makes the practical implementation of the MTV procedure in complex geophysical models simpler with the same reduced stochastic equations for the unresolved modes. In their study, a barotropic model with a realistic climate was used to derive low-order stochastic models by the MTV strategy. Low-order models with as little as two resolved modes succeed in capturing the geographical distributions of the climatological mean field, the variance, and the eddy forcing. Furthermore, the envelope of the autocorrelation functions is captured reasonably well.

The present study applies the systematic stochastic mode reduction strategy to a baroclinic three-layer quasigeostrophic model on the sphere (Marshall and Molteni 1993). In section 2 the model and its main climatological statistics are presented. Section 3 outlines the stochastic mode reduction strategy, and section 4 presents results from the reduced stochastic models, while section 5 is devoted to variants of reduced stochastic models without climate drift. This paper closes with a summary and discussion in section 6 .

\section{Prototype atmospheric GCM}

\section{a. Quasigeostrophic model}

The prototype atmospheric GCM is a spectral threelevel quasigeostrophic (QG) model as described in Marshall and Molteni (1993) and appendix A. The QG equation can be written as

$$
\frac{\partial q}{\partial t}=-J(\psi, q)-D(\psi)+S
$$

where $q$ is potential vorticity (PV), $\psi$ streamfunction, $D(\psi)$ a linear operator that represents dissipative terms, $S$ a constant PV source, and $J$ the Jacobian operator. The model is triangularly truncated at total wavenumber 21. The constant forcing $S$ is derived from the European Centre for Medium-Range Weather Forecasts (ECMWF) data in order to simulate the observed climatological mean state and variance [see Marshall and Molteni (1993) for a more detailed discussion]. The model is integrated with a fourth-order Adams-Bashforth numerical scheme with a time step of $10 \mathrm{~min}$. The state vector is saved every $6 \mathrm{~h}$. The model is integrated in hemispheric mode for the North- ern Hemisphere and is started from random initial conditions for 500000 days after skipping the first 10000 days in order to eliminate transient behavior.

\section{b. Climatology}

We will now discuss the climatology of the quasigeostrophic model. As a reference we also provide a climatology of ECMWF data, which has been used to calculate the forcing for the QG model (Marshall and Molteni 1993). The ECMWF data span the time period from 1983 to 1992 for boreal winter at the three pressure levels 200, 500, and $800 \mathrm{hPa}$. The winds are calculated from the streamfunction; therefore, they are nondivergent.

The model reproduces the climatological zonal wind and streamfunction well (Fig. 1a). The Pacific jet exit region is too far upstream and the Atlantic jet is also slightly shifted upstream compared with the ECMWF data (not shown). The bandpass-filtered (periods between 2.5 and 6 days; Blackmon 1976) streamfunction variance is too weak compared with the ECMWF data but the location of the storm tracks is well reproduced (Fig. 1b). The low-pass-filtered (periods greater than 10 days; Blackmon 1976) streamfunction variance is stronger than in the ECMWF data but the locations of the maxima are well reproduced (not shown). To quantify the quality of the geographical distribution of the various fields pattern correlations are calculated between the QG model and ECMWF fields (Table 1). The pattern correlations for the streamfunction mean and standard deviation fields are above 0.93 (Table 1). The mean zonal wind has pattern correlation of 0.91 on the uppermost layer and 0.73 on the lowest layer. Overall, the ECMWF climate is reproduced reasonably well by the QG model and is suitable as a test climate for the stochastic mode reduction strategy.

\section{c. Total energy metric EOFs}

Total energy metric EOFs [see appendix B and Ehrendorfer (2000)] have been computed from the model output. The first EOF explains $7 \%$ and the second $4 \%$ of the total variance. The leading EOFs do not resemble any well-known teleconnection patterns found in observations, but the subset of the first 10 EOFs nonetheless contains good approximations of the observed NAO and PNA patterns. To quantify this, the observed NAO and PNA patterns are derived from 300-hPa streamfunction (Franzke and Feldstein 2005). All patterns are normalized to unit length in a squared streamfunction metric. The observed PNA or NAO, denoted as $g$, can be written in terms of the QG model 
a) QGM

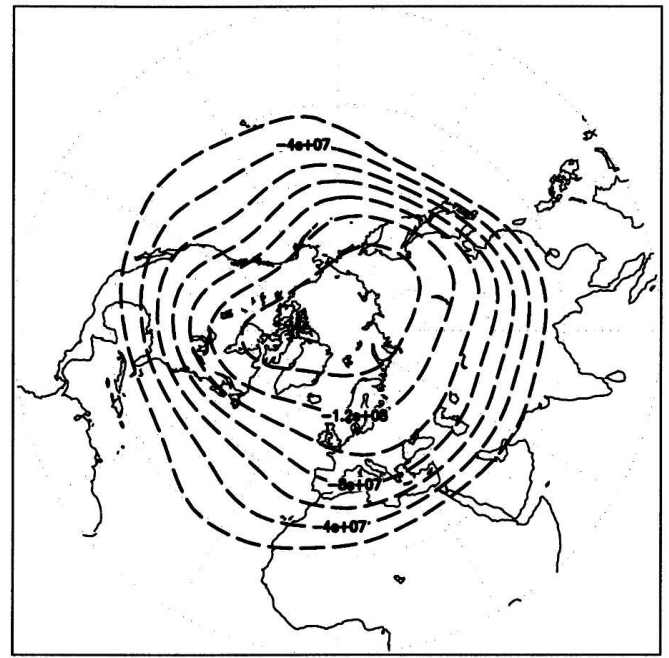

b) QGM

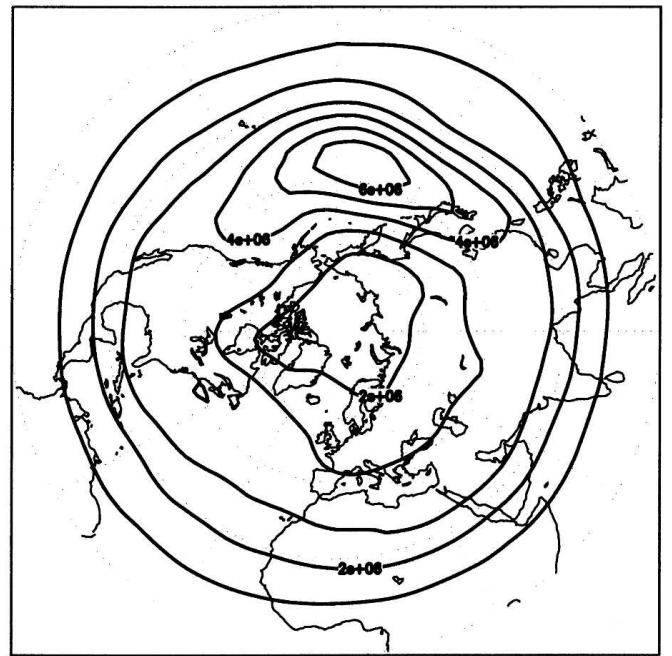

ECMWF

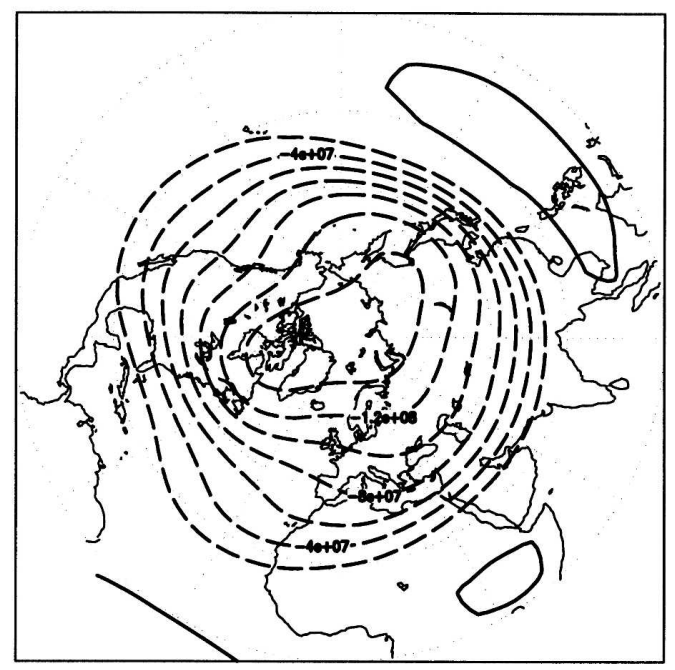

ECMWF

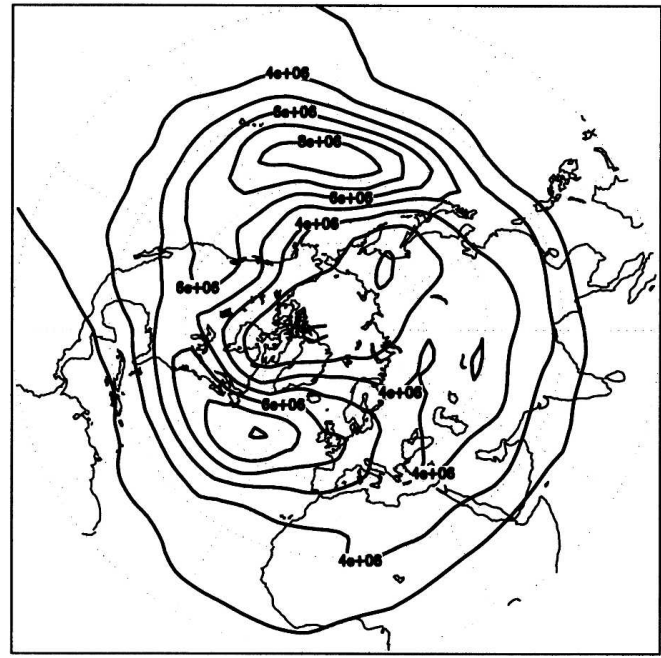

FIG. 1. (a) Climatological streamfunction at $200 \mathrm{hPa}$, and (b) standard deviation of bandpass-filtered streamfunction at $200 \mathrm{hPa}$. Dashed lines indicate negative values.

EOFs as $g=\Sigma_{i} a_{i} e_{i}$, where $e_{i}$ are the QG model EOFs at $200 \mathrm{hPa}$, and $a_{i}$ are scalar constants given by $a_{i}=\int g e_{i}$ $d \Omega$. By considering only a subset of the EOFs, a quantitative measure of how much of the observed NAO or PNA is contained in the subset is given by the pattern correlation between the NAO (PNA) and the subset reconstruction $f=\sum_{i}^{N} a_{i} e_{i}$. Table 2 shows that by increasing the number of retained QG model EOFs the PNA and NAO are increasingly better captured. The first ten EOFs capture roughly $70 \%$ of the PNA and $80 \%$ of the NAO. The first 10 to 20 total energy metric EOFs are nearly equivalent barotropic in the upper two layers, which is quantified by the pattern correlation between the 500- and 200-hPa levels (Fig. 2). Mediumand higher-order EOFs tend to be more baroclinic.

The time scale set by the autocorrelation function is an important measure in this study, because for the development of a stochastic climate model we have to

TABLE 1. Pattern correlations between QG model and ECMWF mean fields.

\begin{tabular}{ccccc}
\hline \hline Level & $\bar{\psi}$ & $\bar{u}$ & $\sqrt{\overline{\psi^{B^{2}}}}$ & $\sqrt{\overline{\psi^{L^{2}}}}$ \\
\hline $200 \mathrm{hPa}$ & 0.99 & 0.91 & 0.95 & 0.98 \\
$500 \mathrm{hPa}$ & 0.98 & 0.86 & 0.94 & 0.97 \\
$800 \mathrm{hPa}$ & 0.95 & 0.73 & 0.93 & 0.96 \\
\hline
\end{tabular}


TABLE 2. Projection of EOF subspace onto observed PNA and NAO patterns from Franzke and Feldstein (2005).

\begin{tabular}{ccc}
\hline \hline Number of EOFs & PNA & NAO \\
\hline 3 & 0.02 & 0.16 \\
4 & 0.25 & 0.24 \\
5 & 0.46 & 0.26 \\
6 & 0.58 & 0.28 \\
7 & 0.62 & 0.42 \\
8 & 0.65 & 0.57 \\
9 & 0.67 & 0.68 \\
10 & 0.68 & 0.78
\end{tabular}

make the truncation between slow and fast modes. The autocorrelation time scale, as defined as the integral over the absolute value of the autocorrelation function $T_{k}(s)$,

$$
T_{k}(s)=\frac{\left\langle a_{k}(t) a_{k}(t+s)\right\rangle}{\left\langle a_{k}^{2}\right\rangle},
$$

where $k$ indicates the $k$ th EOF mode, $s$ the time lag, and $\langle\cdot\rangle$ denotes a time average; $\int\left|T_{k}(s)\right| d s$ indicates how slow or fast a variable loses its memory. The spectrum of the autocorrelation time scale shows a continuous decay with no clear gap (Fig. 3). The first six EOFs have autocorrelation time scales between 22 and 9 days. A further noticeable feature is that the highest EOF has an autocorrelation time scale of about 35 days. Its horizontal structure is nearly zonally symmetric (not shown) and it explains nearly zero variance, therefore, the long time scale seems to be an artifact of the EOF analysis. The autocorrelation functions show almost no oscillations (Fig. 4). Only mode 5 and 10 exhibit some

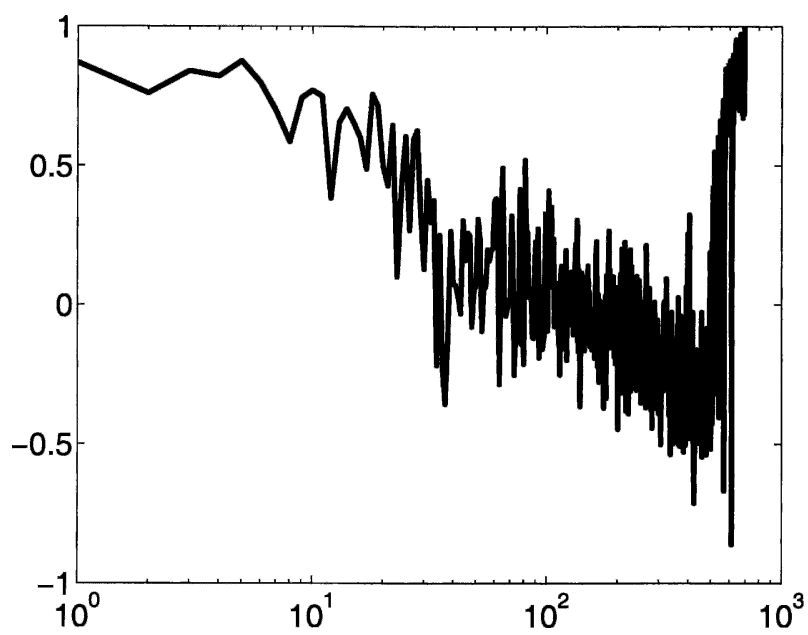

FIG. 2. Semilog plot of the EOF pattern correlation between the 200- and 500-hPa level.

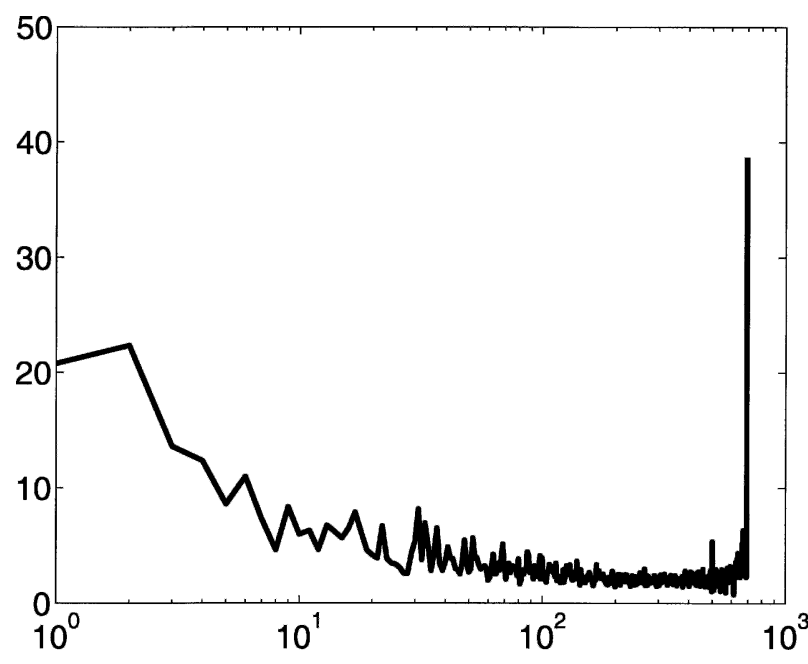

FIG. 3. Autocorrelation time scale of total energy metric EOFs.

modest oscillation. Higher-order moments show only small deviations from Gaussianity (not shown).

An important property of stochastic climate modeling is the separation between slowly evolving lowfrequency (resolved) modes and considerably faster evolving unresolved modes. The stochastic mode elimination procedure (MTV01) is rigorously valid in the limit that the ratio of the autocorrelation time scale of the slowest unresolved mode to the fastest resolved modes goes to zero. Several examples from MTV02 and MTV03 show that for ratios of up to 0.5 , and sometimes even 1.0, this approach is still applicable. For the cases presented here this ratio is relatively large; in particular, by considering only the first four modes as resolved this ratio is 0.6 , for the first six modes as climate modes this ratio becomes 0.8 , and for even more resolved modes this ratio is getting close to one. As shown below, despite these large ratios the stochastic low-order models perform reasonably well. Also the study by Franzke et al. (2005) showed that for even a large ratio the MTV procedure performs reasonably well for a realistic barotropic model climate.

\section{Systematic stochastic mode reduction}

We illustrate the ideas for stochastic climate modeling for the quasigeostrophic model (1), which we write in the following symbolic form:

$$
\frac{\partial u}{\partial t}=F+L u+B(u, u),
$$

where $u$ denotes the state vector with the basis state subtracted. Basic-state effects are included in the definitions of $F$ and $L$ (Franzke et al. 2005).

In stochastic climate modeling, the variable $u$ is de- 
a)

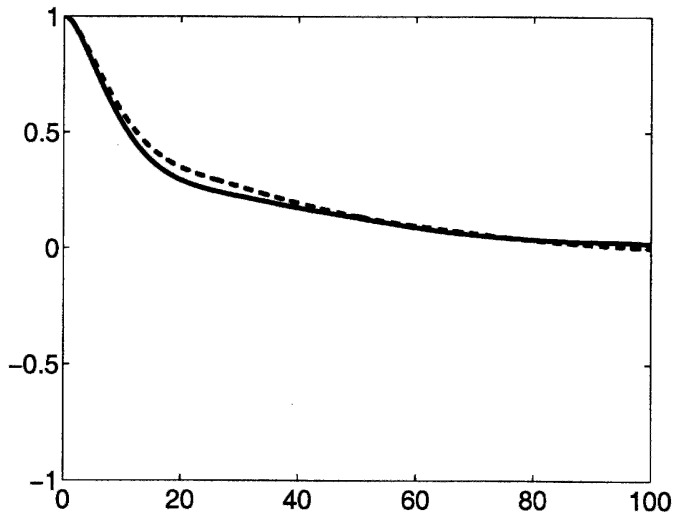

c)

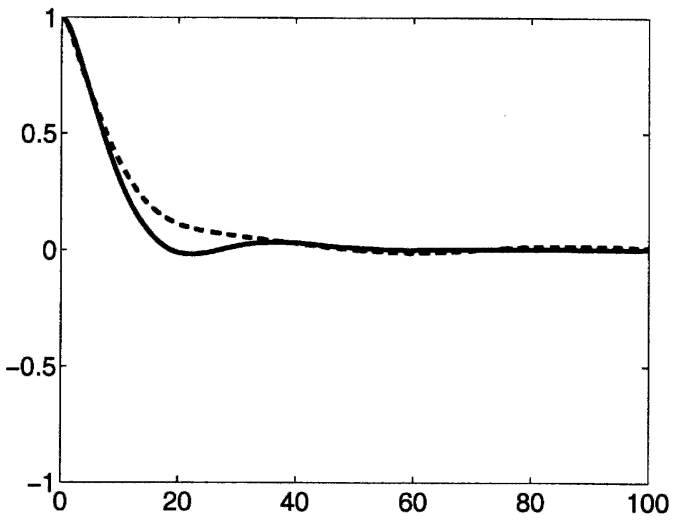

e)

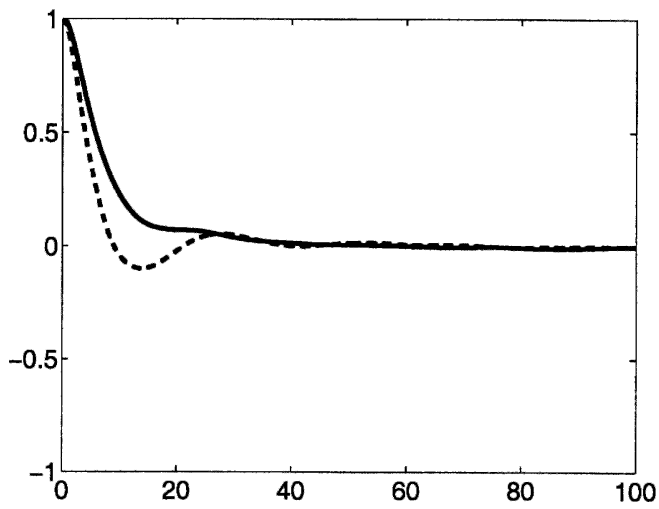

b)

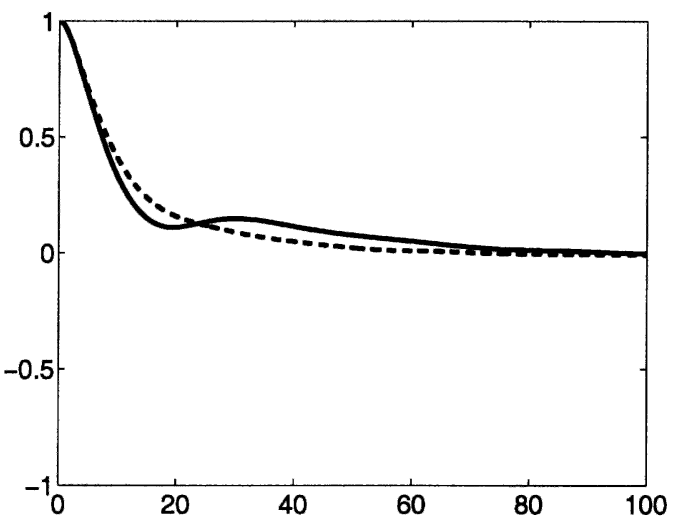

d)

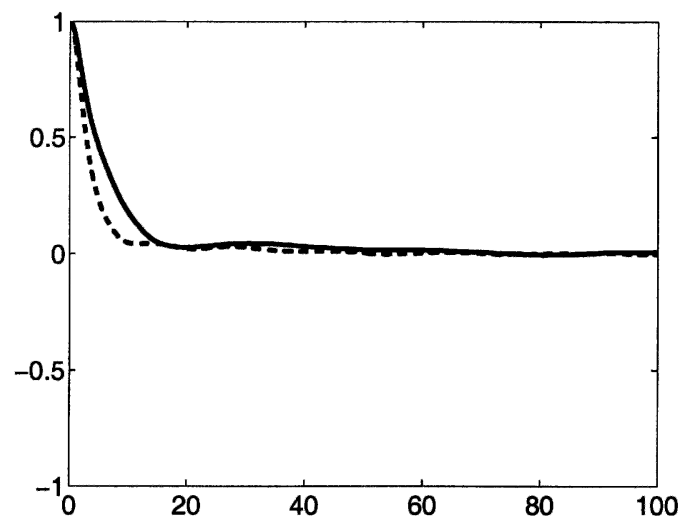

FIG. 4. Autocorrelation function: (a) mode 1 (solid line) and mode 2 (dashed line), (b) mode 3 (solid line) and mode 4 (dashed line), (c) mode 5 (solid line) and mode 6 (dashed line), (d) mode 7 (solid line) and mode 8 (dashed line), (e) mode 9 (solid line) and mode 10 (dashed line).

composed into an orthogonal decomposition through the variables $\tilde{u}$ and $u^{\prime}$, which are characterized by strongly differing time scales. The variable $\tilde{u}$ denotes a low-frequency mode of the system, which evolves slowly in time compared to the $u^{\prime}$ variables. By decomposing $u=\tilde{u}+u^{\prime}$ in terms of total energy metric EOFs $e_{i}$ (appendix $\mathrm{B}$ ) we can write them as

$$
u=\sum_{i=1}^{N} a_{i} e_{i}=\sum_{i=1}^{R} \alpha_{i} e_{i}+\sum_{j=R+1}^{N} \beta_{j} e_{j},
$$

with $\tilde{u}=\sum_{i=1}^{R} \alpha_{i} e_{i}$, and $u^{\prime}=\sum_{j=R+1}^{N} \beta_{j} e_{j}$, where $R$ is the number of resolved modes, $a_{i}$ denote the EOF expansion coefficients, $\alpha_{i}\left(\beta_{j}\right)$ are the expansion coefficients of the resolved (unresolved) modes, and $N=693$ for the present application. The use of the total energy metric ensures the conservation of total energy by the nonlinear operator and prevents nonlinear instabilities (Achatz and Branstator 1999). By properly projecting the EOF expansions onto Eq. (3), we get two sets of equations for resolved $\alpha_{i}$ and unresolved $\beta_{i}$ modes: 


$$
\begin{aligned}
\dot{\alpha}_{i}(t)= & H_{i}^{\alpha}+\sum_{j} L_{i j}^{\alpha \alpha} \alpha_{j}(t)+\frac{1}{\varepsilon} \sum_{j} L_{i j}^{\alpha \beta} \beta_{j}(t) \\
& +\sum_{j k} B_{i j k}^{\alpha \alpha \alpha} \alpha_{j}(t) \alpha_{k}(t) \\
& +\frac{2}{\varepsilon} \sum_{j k} B_{i j k}^{\alpha \alpha \beta} \alpha_{j}(t) \beta_{k}(t)+\frac{1}{\varepsilon} \sum_{j k} B_{i j k}^{\alpha \beta \beta} \beta_{j}(t) \beta_{k}(t)
\end{aligned}
$$

$$
\begin{aligned}
\dot{\beta}_{i}(t)= & H_{i}^{\beta}+\frac{1}{\varepsilon} \sum_{j} L_{i j}^{\beta \alpha} \alpha_{j}(t)+\frac{1}{\varepsilon} \sum_{j} L_{i j}^{\beta \beta} \beta_{j}(t) \\
& +\frac{1}{\varepsilon} \sum_{j k} B_{i j k}^{\beta \alpha \alpha} \alpha_{j}(t) \alpha_{k}(t) \\
& +\frac{2}{\varepsilon} \sum_{j k} B_{i j k}^{\beta \alpha \beta} \alpha_{j}(t) \beta_{k}(t)+\frac{1}{\varepsilon^{2}} \sum_{j k} B_{i j k}^{\beta \beta \beta} \beta_{j}(t) \beta_{k}(t),
\end{aligned}
$$

where the interaction coefficients are defined in appendix $\mathrm{B}$, and the nonlinear operators have been symmetrized, that is, $B_{i j k}=B_{i k j}$ in (5). The upper indices $\alpha$ and $\beta$ indicate the respective subsets of the full operators in (3). Here $\varepsilon$ is a small positive parameter that controls the separation of time scale between slow and fast modes and measures the ratio of the correlation time of the slowest unresolved mode $u^{\prime}$ to the fastest resolved mode $\tilde{u}$. In placing the parameter in front of particular terms we tacitly assume that they evolve on a faster time scale then the terms involving the resolved modes alone. Ultimately, $\varepsilon$ is set to the value $\varepsilon=1$ in developing all the final results (MTV02; MTV03), that is, introducing $\varepsilon$ is only a technical step in order to carry out the MTV mode reduction strategy. Such a use of $\varepsilon$ has been checked on a wide variety of idealized examples where the actual value of $\varepsilon$ ranges from quite small to order one (MTV02; MTV03; Majda and Timofeyev 2004; Majda et al. 2005). In particular, in (5) we make the following assumptions:

- $H^{\alpha}, H^{\beta}, L^{\alpha \alpha}$, and $B^{\alpha \alpha \alpha}$ act on a time scale of order one.

- The linear operators $L^{\alpha \beta}, L^{\beta \alpha}$, and $L^{\beta \beta}$ act at most on a faster time scale of order $\varepsilon^{-1}$.

- The self-interaction of the unresolved modes $B^{\beta \beta \beta}$ acts on the fastest time scale of order $\varepsilon^{-2}$, whereas, $B^{\alpha \alpha \beta}, B^{\alpha \beta \beta}, B^{\beta \alpha \alpha}$, and $B^{\beta \alpha \beta}$ act on a time scale of order $\varepsilon^{-1}$.

Furthermore, it is assumed that the dynamical system given only by unresolved modes with interaction $B^{\beta \beta \beta}$ is ergodic and mixing, and can be represented by a stochastic process, and that all unresolved modes are quasi Gaussian distributed (based on the model dynamics, this assumption is valid). With these assumptions, the MTV procedure (Franzke et al. 2005) predicts the functional form of the following effective stochastic equations for the resolved variables alone:

$$
\begin{aligned}
d \alpha_{i}(t)= & H_{i}^{\alpha} d t+\sum_{j} L_{i j}^{\alpha \alpha} \alpha_{j}(t) d t+\sum_{j k} B_{i j k}^{\alpha \alpha \alpha} \alpha_{j}(t) \alpha_{k}(t) d t \\
& +\tilde{H}_{i} d t+\sum_{j} \tilde{L}_{i j} \alpha_{j}(t) d t \\
& +\sum_{j k} \tilde{B}_{i j k} \alpha_{j}(t) \alpha_{k}(t) d t \\
& +\sum_{j k l} \tilde{M}_{i j k l} \alpha_{j}(t) \alpha_{k}(t) \alpha_{l}(t) d t \\
& +\sqrt{2} \sum_{j} \sigma_{i j}^{(1)}(\alpha(t)) d W_{j}^{(1)}+\sqrt{2} \sum_{j} \sigma_{i j}^{(2)} d W_{j}^{(2)}
\end{aligned}
$$

This stochastic differential equation system is in Itô form (Gardiner 1985). This system consists of the bare truncation, deterministic correction terms as well as additive and multiplicative noises. The correction terms and noises are predicted by the systematic stochastic mode reduction strategy and account for the interaction between resolved and unresolved modes, as well as for the self-interaction between the unresolved modes. The explicit values of the coefficients in (6a) are given by the appropriate subsets of the interaction coefficients of appendix B, whereas, the coefficients in (6b)-(6c) are explicitly determined by the seamless MTV procedure in Franzke et al. (2005) and appendix C with the only inputs from the unresolved modes involving their variances and the integrated autocorrelation components (the unresolved correlation times).

To see which of these correction terms play a vital role in the integrations of the low-order stochastic model (6) we group the interaction terms between resolved and unresolved modes according to their physical origin and set a parameter $\lambda_{i}$ in front of the corresponding interaction coefficient. The bare truncation is indicated by a $\lambda_{B}$ and describes the interaction between the resolved modes. The interaction between the triads $B^{\alpha \beta \beta}$ and $B^{\beta \alpha \beta}$ gives rise to additive noise and a linear correction term, and describes the driving of the resolved modes by the unresolved ones; we will name these triads additive triads and set a $\lambda_{A}$ in front of them (MTV99; MTV01; MTV02; MTV03). The second type of triad interaction is between $B^{\alpha \alpha \beta}$ and $B^{\beta \alpha \alpha}$. These interactions create multiplicative noises and cubic nonlinear correction terms (MTV99; MTV01; MTV02; 
MTV03); we will call them multiplicative triads in the following and indicate them by a $\lambda_{M}$. These triad interactions describe the advection of resolved modes by the unresolved modes, which induce tendencies in the resolved modes. The linear coupling between the resolved and unresolved modes $L^{\alpha \beta}$ and $L^{\beta \alpha}$, give rise to additive noise and a linear correction term (MTV01; Franzke et al. 2005), which is called the augmented linearity here and is indicated by a $\lambda_{L}$. The augmented linearity describes the effect of the linear interaction between the unresolved (resolved) modes and the climatological mean state onto the resolved (unresolved) modes. We set a $\lambda_{F}$ in front of the last remaining interaction term $L^{\beta \beta}$, the linear coupling of the unresolved modes. The quadratic nonlinear corrections, a forcing term and a further multiplicative noise contribution are caused by the interaction between the linear coupling terms and the multiplicative triads. Another forcing correction term comes from the interaction between additive triads and the linear coupling of the fast modes.

After regrouping the terms the stochastic climate model from (6) can be written according to these physical processes as

$$
\begin{aligned}
d \alpha_{i}(t)= & \lambda_{B}\left[H_{i}^{\alpha} d t+\sum_{j} L_{i j}^{\alpha \alpha} \alpha_{j}(t) d t\right. \\
& \left.+\sum_{j k} B_{i j k}^{\alpha \alpha \alpha} \alpha_{j}(t) \alpha_{k}(t) d t\right] \\
& +\lambda_{A}^{2} \sum_{j} \tilde{L}_{i j}^{(2)} \alpha_{j}(t) d t+\lambda_{A} \sqrt{2} \sum_{j} \sigma_{i j}^{(2)} d W_{j}^{(2)} \\
& +\lambda_{M}^{2}\left[\sum_{j} \tilde{L}_{i j}^{(3)} \alpha_{j}(t) d t+\sum_{j k l} \tilde{M}_{i j k l} \alpha_{j}(t) \alpha_{k}(t) \alpha_{l}(t) d t\right] \\
& +\lambda_{L}^{2}\left[\sum_{j} \tilde{L}_{i j}^{(1)} \alpha_{j}(t) d t\right] \\
& +\lambda_{M} \lambda_{L}\left[\tilde{H}_{j}^{(1)} d t+\sum_{j k} \tilde{B}_{i j k} \alpha_{j}(t) \alpha_{k}(t) d t\right] \\
& +\lambda_{A} \lambda_{F} \tilde{H}_{j}^{(2)} d t \\
& +\sqrt{2} \sum_{j} \sigma_{i j}^{(1)}[\alpha(t)] d W_{j}^{(1)},
\end{aligned}
$$

where the nonlinear noise matrix $\sigma^{(1)}$ satisfies

$$
\begin{gathered}
\lambda_{L}^{2} Q_{i j}^{(1)}+\lambda_{L} \lambda_{M} \sum_{k} U_{i j k} \alpha_{k}(t)+\lambda_{M}^{2} \sum_{k l} V_{i j k l} \alpha_{k}(t) \alpha_{l}(t) \\
=\sum_{k} \sigma_{i k}^{(1)}[\alpha(t)] \sigma_{j k}^{(1)}[\alpha(t)] .
\end{gathered}
$$

TABLE 3. Dynamical behavior of the bare truncation models.

\begin{tabular}{cl}
\hline Number of modes & \multicolumn{1}{c}{ Behavior } \\
\hline 2 & One fixed point \\
3 & One fixed point \\
4 & One fixed point \\
5 & One fixed point \\
6 & One stable periodic orbit \\
7 & One fixed point \\
8 & Mixing \\
9 & Mixing \\
10 & One stable periodic orbit \\
\hline
\end{tabular}

It is guaranteed (MTV01) that the operator on the lefthand side of (8) is always positive definite ensuring the existence of the nonlinear noise matrix on the righthand side. In general, the equation in (8) determines a multiplicative noise operator. By considering only contributions from the linear coupling, in other words, setting $\lambda_{M}=0$ in (8), this multiplicative noise operator reduces to an additive noise operator, which comes from the linear coupling between resolved and unresolved modes. All coefficients are defined explicitly in appendix $\mathrm{C}$.

For integrating this stochastic differential equation a split-step numerical procedure is used with a fourthorder Runge-Kutta scheme for the deterministic part and an Euler-forward scheme for the stochastic part. A time step size of 0.01 days has been used for all integrations. Statistics of the stochastic model are calculated by time averaging an individual solution integrated over a long time of the order of $10^{6}$ days.

\section{Bare truncation results}

First we discuss the dynamical behavior of low-order bare truncation model integrations with different numbers of retained EOFs. For doing this we set $\lambda_{B}=1$ and $\lambda_{A}=\lambda_{L}=\lambda_{M}=\lambda_{F}=0$ in (7). Therefore, the bare truncation models do not account for the unresolved modes. The bare truncation models are integrated for $10^{4}$ days starting from $10^{4}$ random initial conditions drawn from a normal distribution. This is done to see if the bare truncation models either go to a fixed point (and if it is always the same fixed point), into a periodic orbit or exhibit chaotic mixing behavior. The bare truncation models with two, three, four, five, and seven modes go always to the same fixed point (Table 3 ). The 8- and 9-mode bare truncation models are mixing (Table 3), whereas, the models with 6 and 10 retained modes exhibit the oscillatory behavior of a stable periodic orbit as can be seen in state space projections of the EOF modes for the 10-mode system (Fig. 5a). 
a)

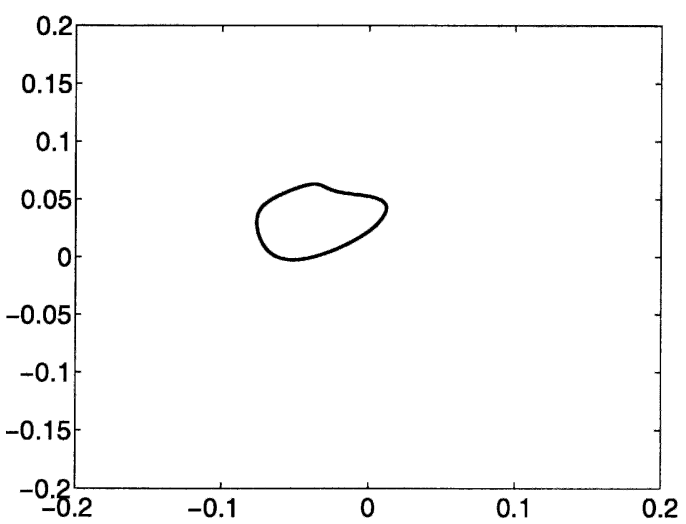

b)

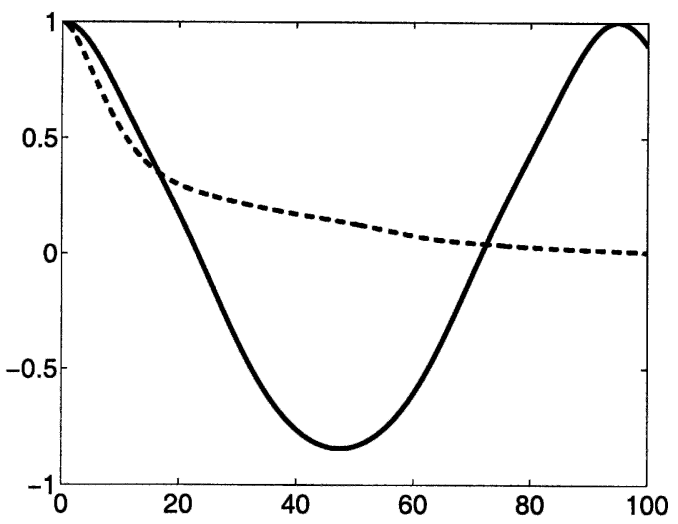

FIG. 5. (a) Ten-mode bare truncation integration from $10^{4}$ different initial conditions (showed is the projection onto the EOF2 and EOF5 plane), (b) autocorrelation function of mode 1 of the 10-mode bare truncation integration (bare truncation: solid line, QG model: dashed line).

Furthermore, an individual long time integration of the 10-mode system shows a huge climate drift, too much variance and the autocorrelation function confirms the strong oscillatory behavior (Fig. 5b). These results indicate that the bare truncation does not capture the essential dynamics. Long time integrations of the four-, six-, and eight-mode systems show qualitatively similar behavior (not shown).

\section{Results of stochastic mode reduction}

\section{a. Integration of the complete MTV stochastic model}

Now we apply the systematic stochastic mode reduction strategy to see how the predicted deterministic correction terms and noises improve the results compared with the bare truncation results of the previous section. First we will present results from a simplified framework, which requires the least input of information from the unresolved modes where we assume that all cross correlations are zero between the unresolved modes. This means that we set $\mathcal{B}_{i j}(t)=\mathcal{B}_{i}(t) \delta_{i j}$ in Eqs. (C1)-(C16). Long time integrations of $10^{6}$ days have been carried out for low-order stochastic models with only $4,6,8$, and 10 resolved modes. In the following we will primarily concentrate on the ten-mode system. We will refer to this run as systematic MTV (SMTV). Qualitatively similar results have been found for the other truncations.

To compare geographical distributions the $\mathrm{QG}$ model fields are reconstructed from the same number of EOFs as the corresponding stochastic model has resolved modes. The geographical distributions of the standard deviations have high pattern correlation val- ues for all truncations of 0.97 or higher (Table 5), which is also confirmed by a visual inspection of the actual fields (Fig. 6). They only underestimate the amplitudes by a factor of about 1.5. The geographical distributions of the time mean transient eddy forcing $-\nabla^{-2}\left(\boldsymbol{\nabla} \cdot \overline{\mathbf{u}^{\prime} \zeta^{\prime}}\right)$ are reasonably similar; the pattern correlations are high (Table 5), which is confirmed by a visual inspection of the fields (Fig. 7). However, the stochastic model underestimates the amplitude of the eddy forcing by a factor of about 2 in the Atlantic and by a factor of about 6 in the Pacific region.

The stochastic models reproduce the autocorrelation functions reasonably well (Figs. 8). The rate of decay for the first 20 lags are usually well captured. Modes 2 and 3 decay too fast for larger lags, and mode 5 too slow. Modes 6 and 9 exhibit a bump around lag 20 days that is not present in the QG model, and mode 10 misses a bump of the QG model but captures the envelope of the decay, which can be seen in a semilog plot (not shown). Overall the stochastic model captures the autocorrelation function reasonably well. Also the probability density functions (PDF) are well captured overall (Figs. 9). The variances of some modes are underestimated by less than a factor of about 2, but for most modes the variances are well captured.

The individual modes of the various truncated stochastic models have means of the order of about 0.01 (Table 4). Including the MTV correction terms reduced the means significantly overall compared to the corresponding bare truncation integration, even though the means increased of modes 9 and 10 in the stochastic model. The geographical distributions of the means show that the means of the individual modes are climatologically relevant (Fig. 10). The geographical distri- 
a) QG-Model

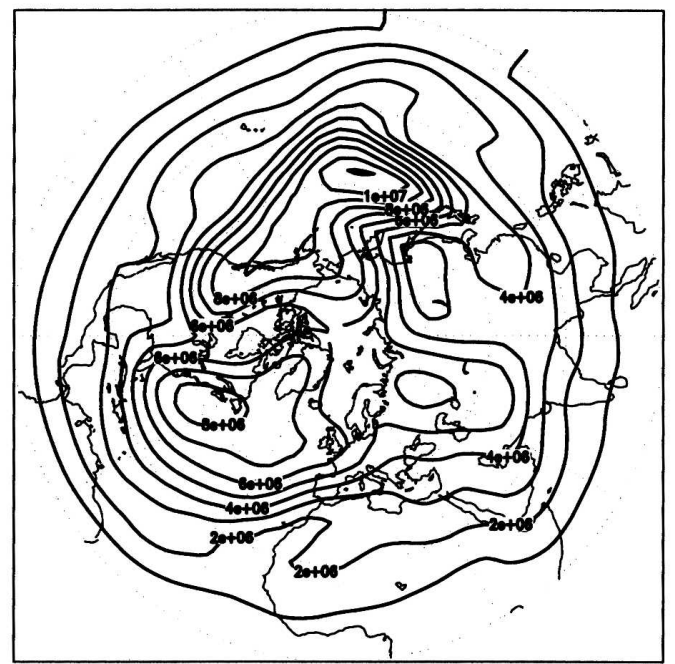

b) S-MTV

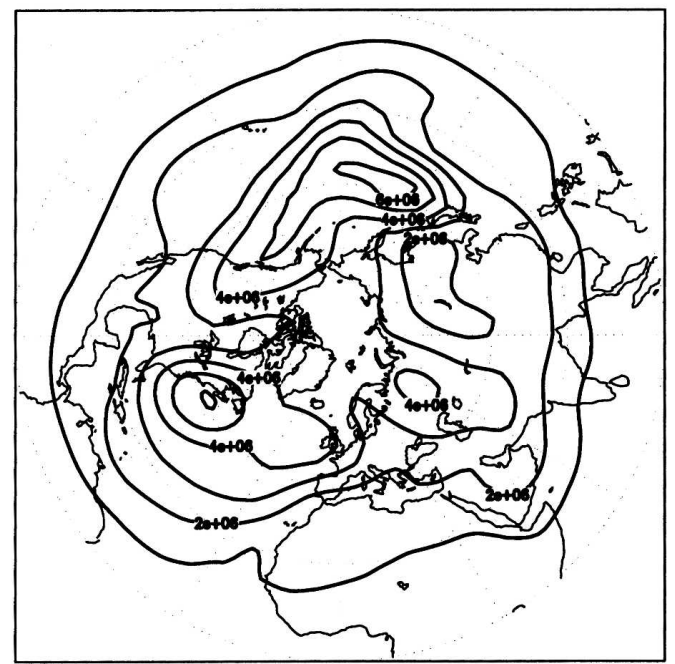

FIG. 6. Climatological streamfuction standard deviation fields composed of the first $10 \mathrm{EOFs}$ at $200 \mathrm{hPa}$ of (a) the quasigeostrophic model, and (b) stochastic model (SMTV). Contour interval: $10^{6} \mathrm{~m}^{2} \mathrm{~s}^{-1}$. The pattern correlations with the corresponding QG model fields are 0.98 at $200 \mathrm{hPa}$ and 0.98 at $500 \mathrm{hPa}$.

bution of the means of four-, six-, and eight-mode systems look far more off from the QG model mean (not shown), and have, therefore, very small pattern correlations (Table 5). The geographical distribution of the 10 -mode system has relatively high pattern correlation values with 0.42 at $200 \mathrm{hPa}$, but a visual inspection shows that the stochastic climate model mean state is still relatively wavy (Figs. 10) and misses the strong zonally symmetric structure of the QG model mean state (Fig. 1a). The likely cause of the climate drift is the too-weak magnitude of the eddy fluxes even though they have high geographic pattern correlations with the true eddy fluxes. Stochastic models with no climate drift will be discussed in section 6 . a) QG-Model

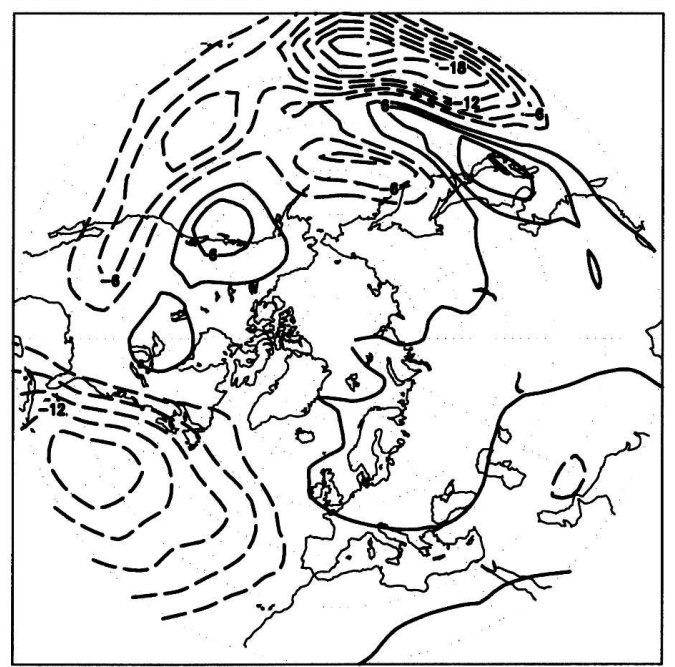

b) S-MTV

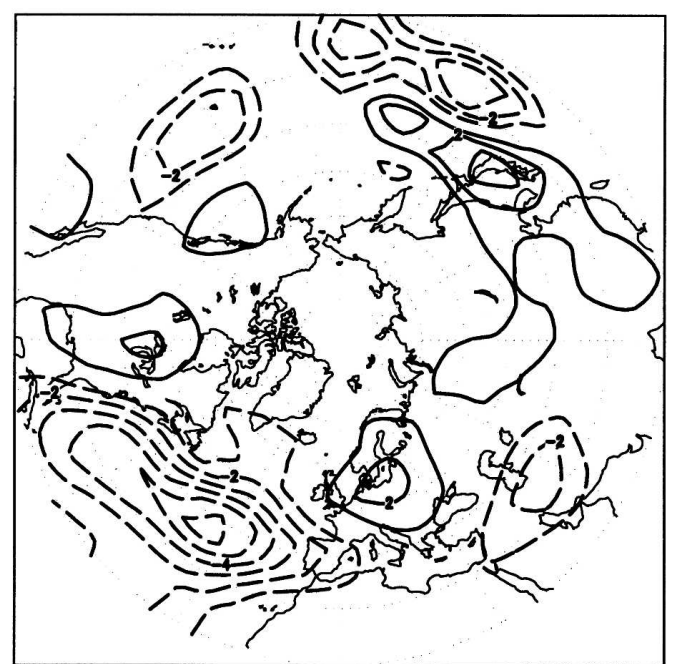

FIG. 7. Climatological eddy forcing fields composed of the first $10 \mathrm{EOFs}$ at $200 \mathrm{hPa}$ of (a) the quasigeostrophic model, and (b) stochastic model (SMTV) eddy forcing. Contour interval: stochastic model $1 \mathrm{~m}^{2} \mathrm{~s}^{-2}$, QG model 3 $\mathrm{m}^{2} \mathrm{~s}^{-2}$, dashed lines indicate negative values. The pattern correlations with the corresponding QG model fields are 0.84 at $200 \mathrm{hPa}$ and 0.74 at $500 \mathrm{hPa}$. 
a)

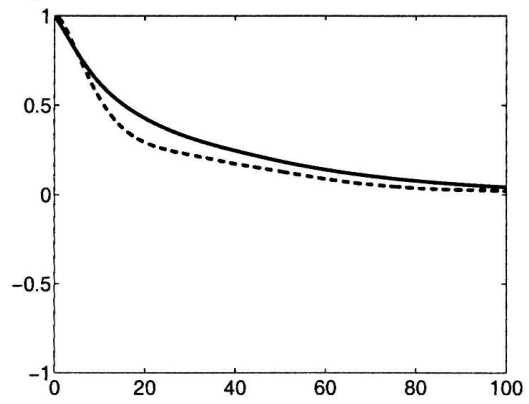

d)

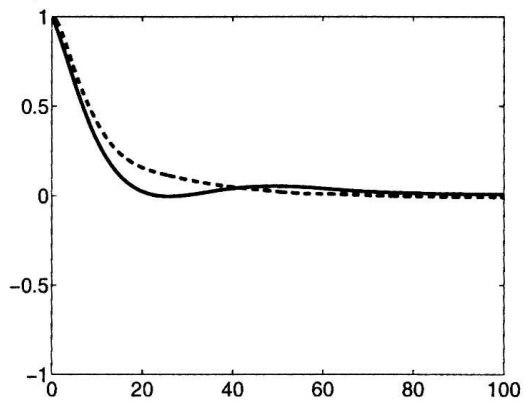

g)

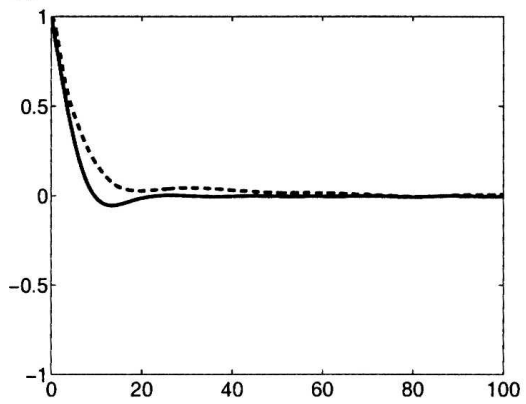

j)

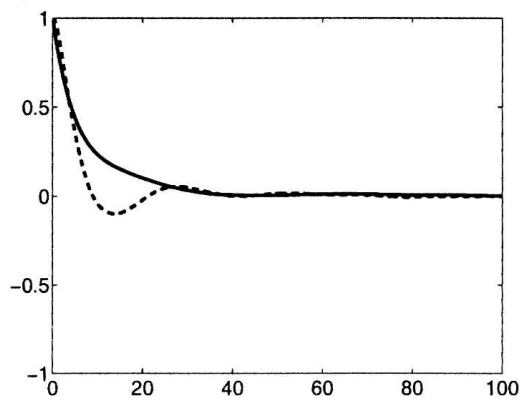

b)

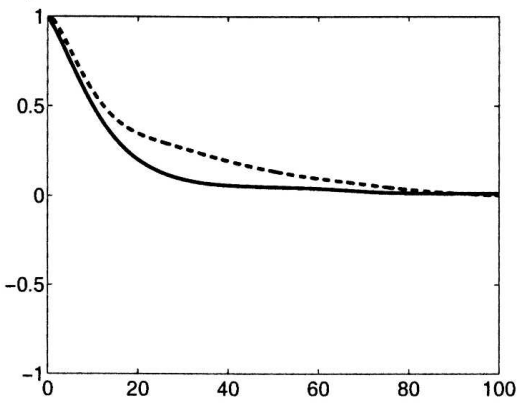

e)

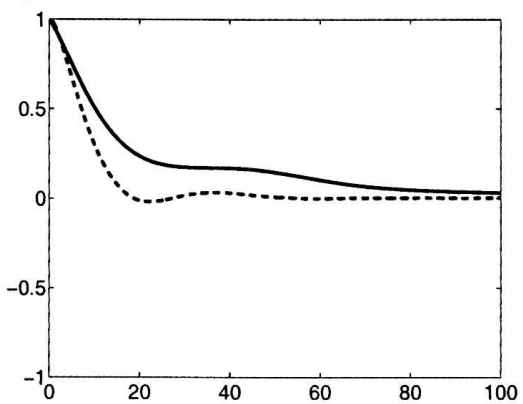

h)

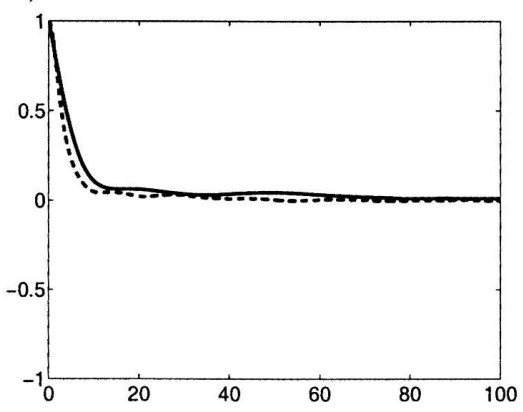

c)

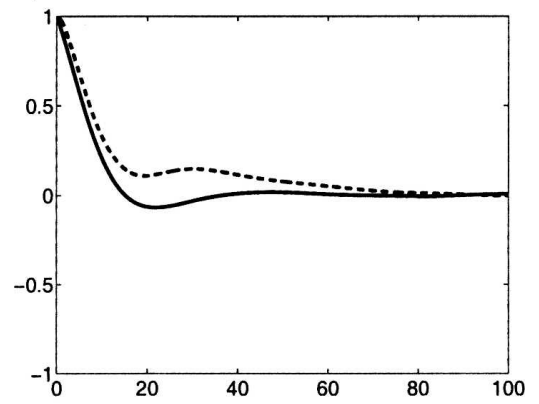

f)

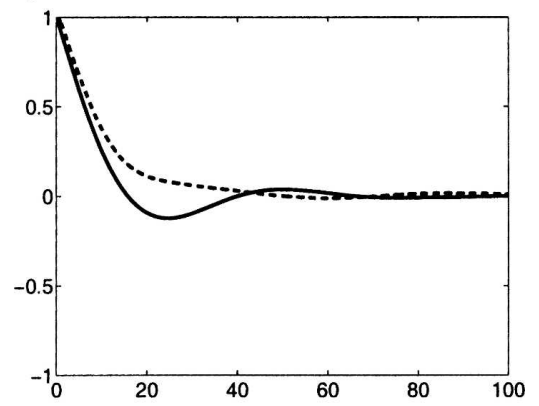

i)

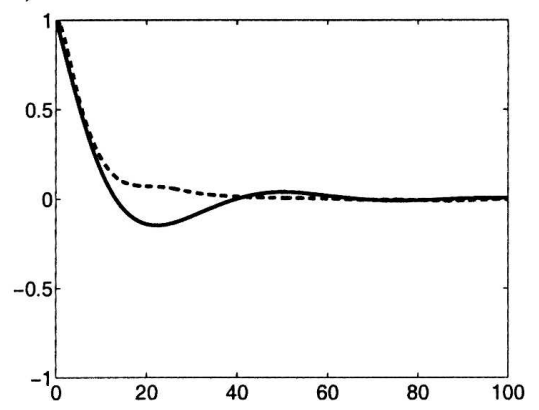

FIG. 8. Autocorrelation function of SMTV: (a) mode 1, (b) mode 2, (c) mode 3, (d) mode 4, (e) mode 5, (f) mode 6, (g) mode 7, (h) mode 8, (i) mode 9, and (j) mode 10 (solid line: stochastic model, dashed line: QG model).

Using the full cross-correlation matrix $B_{i j}(t)$ leads only to minor improvements of the statistics of the stochastic model (not shown). This indicates that the cross correlations are not very important in this model setup.

\section{b. Budget analysis}

The above section shows that the systematic loworder stochastic climate model is performing reason- ably well. This raises the question regarding which of the various correction terms and noises in (7) are important. We will now investigate this question. In Eq. (7) the correction terms are decomposed corresponding to their physical origin. A close investigation of the various correction terms and noises reveals that the bare truncation terms $\left(\lambda_{B}\right)$, the multiplicative triad correction terms $\left(\lambda_{M}\right)$, and the augmented linearity $\left(\lambda_{L}\right)$, 
a)

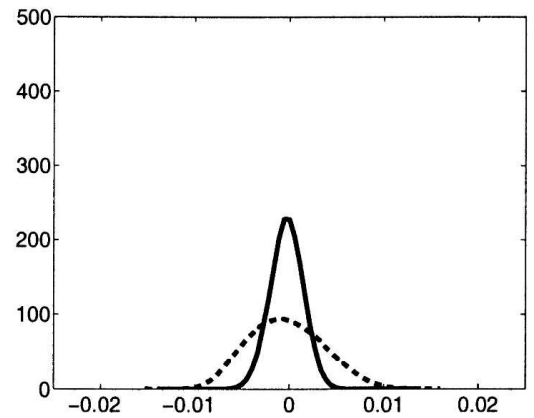

d)

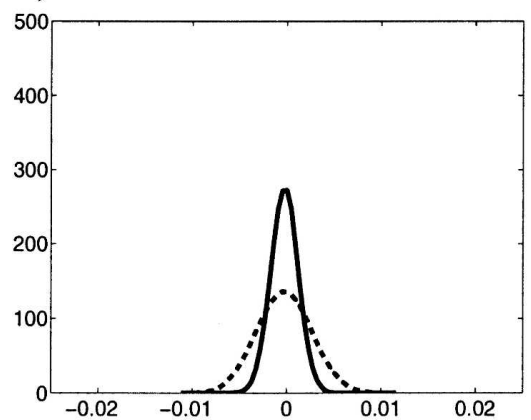

g)

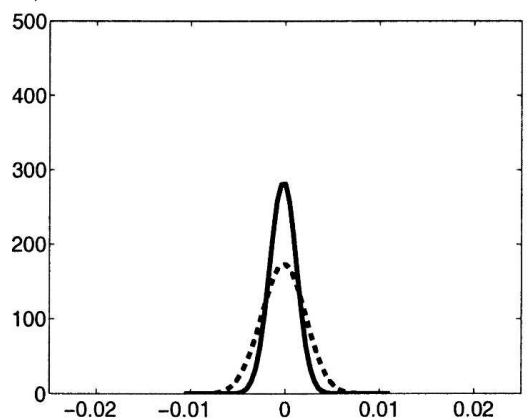

j)

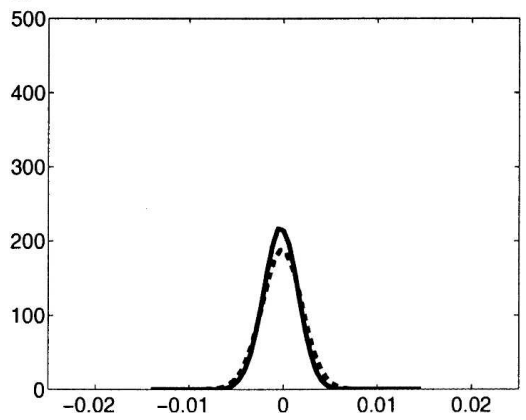

b)

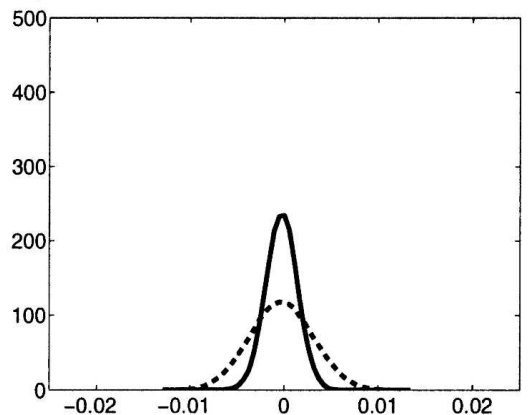

e)

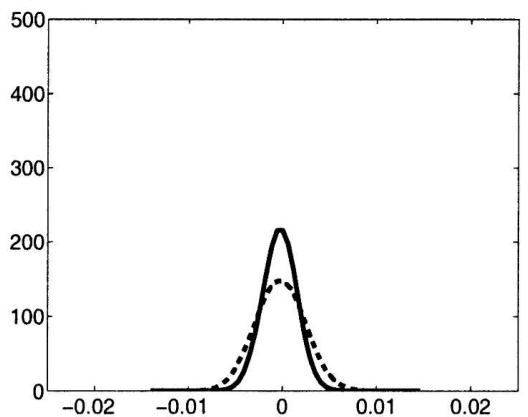

h)

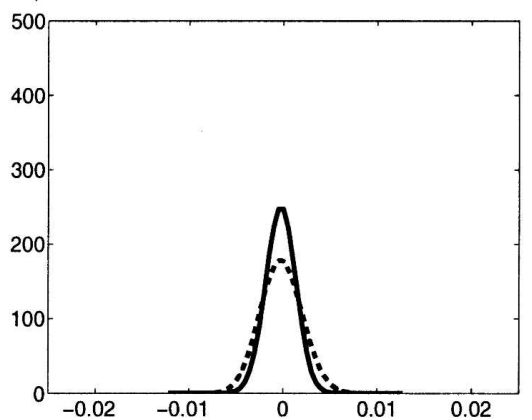

c)

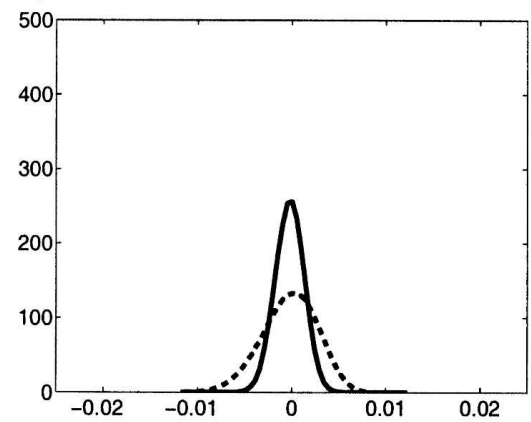

f)

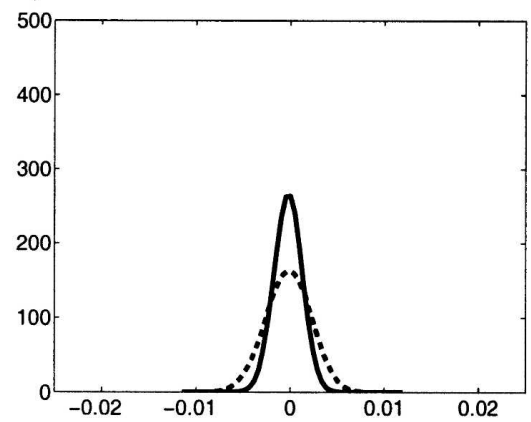

i)

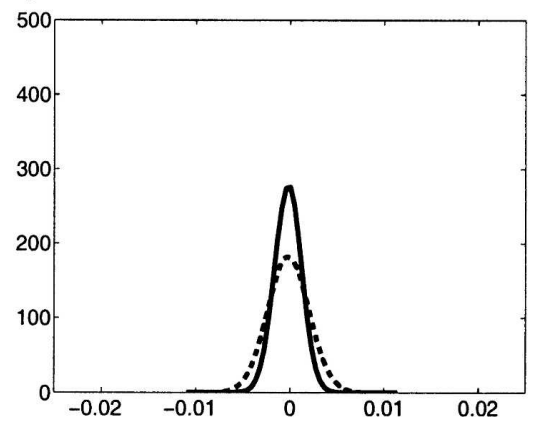

FIG. 9. PDF of SMTV: (a) mode 1, (b) mode 2, (c) mode 3, (d) mode 4, (e) mode 5, (f) mode 6, (g) mode 7, (h) mode 8, (i) mode 9, and (j) mode 10 (solid line: stochastic model, dashed line: QG model).

together with their associated additive and multiplicative noise terms, produce the largest tendencies. The tendencies coming from the remaining correction terms and noises are at least an order of magnitude smaller during the integration of the model.

For this minimal stochastic model (MMTV), which is constructed by setting $\lambda_{A}$ and $\lambda_{F}$ in (7) to zero, the pattern correlation at the $200-\mathrm{hPa}$ level with the QG model mean increases to 0.57 , stays for the standard deviation at 0.98 , but decreases a little bit to 0.74 for the transient eddy forcing compared with SMTV (Table 6). The autocorrelation functions are nearly indistinguish- 
TABLE 4. Means for total energy norm EOFs of reduced models with 10 resolved modes.

\begin{tabular}{crr}
\hline Mode & Bare truncation & SMTV \\
\hline 1 & 0.03 & 0.02 \\
2 & -0.03 & -0.02 \\
3 & 0.02 & -0.02 \\
4 & 0.05 & 0.04 \\
5 & 0.03 & 0.02 \\
6 & -0.07 & -0.03 \\
7 & 0.02 & 0.01 \\
8 & -0.01 & -0.00 \\
9 & -0.01 & -0.04 \\
10 & -0.00 & 0.03 \\
\hline
\end{tabular}

able from the SMTV integration (Figs. 11). Overall this minimal stochastic model reproduces the main features of the SMTV integration well but still has climate drift.

The budget analysis shows the importance of the multiplicative triad interaction $\left(\lambda_{M}\right)$ and of the linear interaction between resolved and unresolved modes $\left(\lambda_{L}\right)$. These interaction terms lead to deterministic linear and nonlinear correction terms and also to additive and multiplicative noises. Nonlinear terms and multiplicative noises have been neglected in previous stochastic modeling studies of midlatitude low-frequency variability (Newman et al. 1997; Whitaker and Sardeshmukh 1998; Zhang and Held 1999; Winkler et al. 2001). The multiplicative noise stems from the advection of the resolved modes by the unresolved modes and the additive noise comes from the projection of the resolved modes onto the linear interaction of the unresolved modes with the climatological mean flow. In previous studies (e.g., Delsole 2004) it has been argued that the additive noise accounts for the neglected nonlinear terms. As the systematic stochastic mode reduction strategy (section 3 ) shows, the only nonlinearities that give rise to additive noise are the additive triad interactions. The systematic strategy has shown that for the 10-dimensional subspace in the present model, derived from total energy metric EOFs, these interactions play only a minor role.

\section{Stochastic mode reduction without climate drift}

The previous section showed that the reduced stochastic models succeed in reproducing the geographical distributions of the standard deviation and of the eddy forcing; also the autocorrelation functions are well captured and the stochastic mode reduction strategy only underestimates the magnitude of the standard deviations slightly. But the reduced stochastic models experience climate drift. This section is devoted to deriving low-order stochastic models without climate drift.

\section{a. MTV stochastic climate model without bare truncation}

A comparison between the bare truncation model integrations (section 4) and the stochastic model integrations (section 5) suggests that the climate drift likely stems from the bare truncation, since the MTV correction terms only somewhat reduce the huge bare truncation climate drift. This raises the question of how well a stochastic model without the bare truncation would reproduce the QG model statistics. This amounts to setting $\lambda_{B}=0$ and $\lambda_{A}=\lambda_{F}=0$ in (7) and is referred to as MRMTV-1 for minimal regression fit MTV. By doing such an integration, it is found that no climate drift occurs; the climatological mean state is nearly perfectly reproduced as is quantified by a pattern correlation value of 0.99 (Table 6). That the climatological mean fields are reproduced with high accuracy is further confirmed by the fact that the means of the individual modes of the stochastic model are very small, of the order of about $\sim 10^{-7}$. Therefore, the individual modes of the MRMTV-1 model reproduce the means of the total energy metric EOFs (which are by construction zero, since we subtracted the time mean) with high accuracy. Also the geographical distribution of the standard deviation is well captured, which can be seen from a pattern correlation value of about 0.90 (Table 6). The eddy forcing is less well reproduced with a pattern correlation value of only 0.35 (Table 6). But the autocorrelation function decays much too slowly (not shown). The MTV stochastic mode reduction strategy leaves one free parameter: the time-scale separation $\varepsilon$. Changing this parameter amounts to a coarse-grained rescaling of time. By setting $\lambda_{M}=\lambda_{L}=4.0$, we rescale time, and the stochastic model (without the bare truncation) captures the time scale and autocorrelation functions of the QG model reasonably well (Figs. 12), even though they provide a somewhat less good fit as in the SMTV run, without changing the variance.

\section{b. Stochastic mode reduction with minimal empirical fitting}

Is it possible to further improve the previous results by varying the $\lambda_{i}$ in (7)? To find a possibly better parameter set $\lambda_{i}$ we vary all $\lambda_{i}$ between 0 and 5 with increments of 1 , which will be refined later to increments of the size of 0.1 for certain parameter regimes.

By doing this we find that the contributions from the additive triads $\lambda_{A}$ and the linear coupling of the unresolved modes $\lambda_{F}$ do not improve the results; therefore, we set $\lambda_{A}=\lambda_{F}=0$ in the following. The best empirical parameter set has been found to be $\lambda_{B}=0.1$, and $\lambda_{M}=$ $\lambda_{L}=4.0$, and will be referred to as MRMTV-2. With 
$200 \mathrm{hPa}$

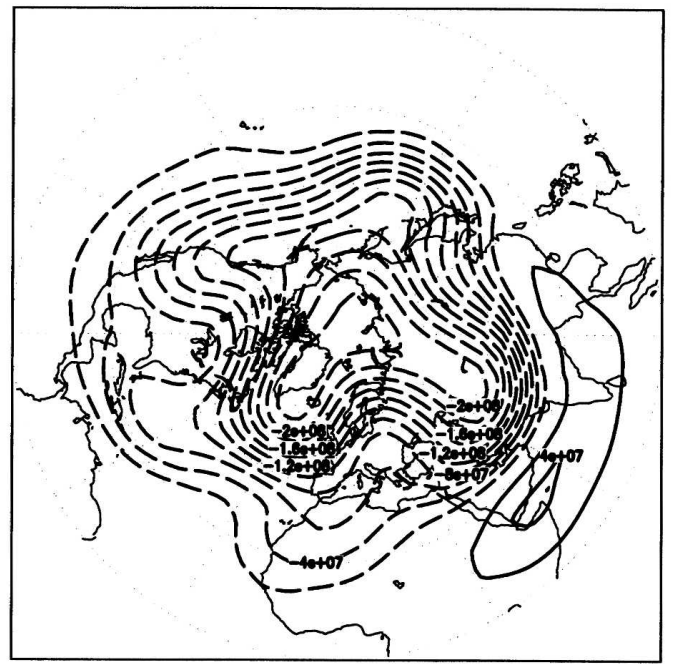

$500 \mathrm{hPa}$

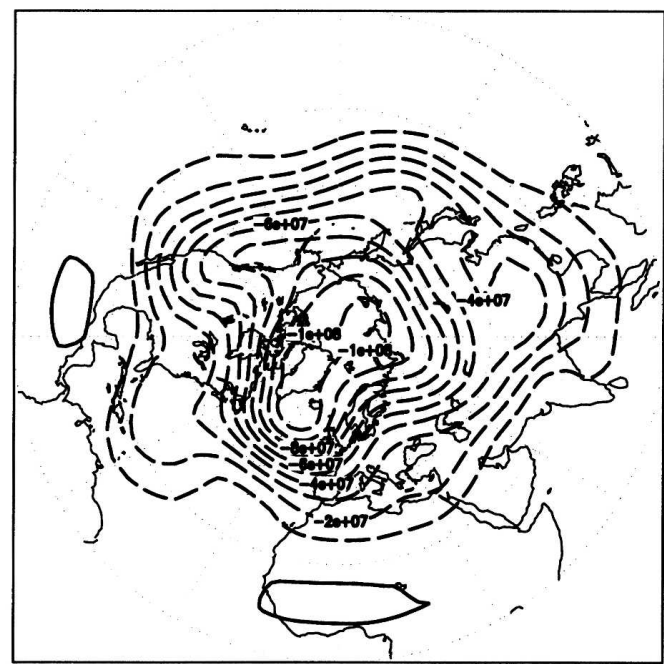

FIG. 10. Mean fields composed of the SMTV run. Contour interval: $200-\mathrm{hPa} 2 \times 10^{7} \mathrm{~m}^{2} \mathrm{~s}^{-1}, 500-\mathrm{hPa} 1 \times 10^{7} \mathrm{~m}^{2}$ $\mathrm{s}^{-1}$, dashed lines indicate negative values. The pattern correlations with the corresponding QG model fields are 0.42 at $200 \mathrm{hPa}$ and 0.43 at $500 \mathrm{hPa}$.

this parameter setup the stochastic model develops no climate drift and as Table 6 shows its geographical distributions of the standard deviation and eddy forcing are better captured than in the earlier case without the bare truncation part. Furthermore, both the autocorrelation functions (Figs. 13) and the PDFs (Figs. 14) are still captured reasonably well. The best parameter fit essentially reduces the strength of the bare truncation on the resolved modes; this reduced coefficient means, intuitively, that we allow the bare truncation terms to act on a longer time scale compared with both the augmented linearity and multiplicative noise corrections that have values increased to 4 . Thus, the very strong climate drift from the bare truncation, reported in section 4 , is minimized by only regression fitting of two parameters.

The previous experiments show that downscaling the bare truncation terms and upscaling the two important MTV terms (augmented linearity and multiplicative triads) gives systematic nonlinear stochastic climate mod- els, which reproduce all main statistical features reasonably well. It also shows that the likely reason for the climate drift lies in the bare truncation terms. As shown in section 4 they do not capture the dynamics and produce a huge climate drift by themselves.

\section{Summary and discussion}

This study applies a systematic strategy for stochastic mode reduction to a prototype atmospheric model with a realistic model climate. The QG model climate used here contains versions of the NAO and PNA teleconnection patterns in its subset of the first ten total energy metric EOFs. The systematic strategy requires first the identification of slowly evolving climate modes and fast evolving nonclimate modes. The low-order stochastic climate model consists of the climate modes as resolved modes and the stochastic mode reduction procedure predicts the functional form of all forcing, linear, quadratic, and cubic correction terms as well as additive

TABLE 5. Pattern correlation between stochastic model (SMTV) and QG model for various truncations: climatological mean, standard deviation, and eddy forcing fields.

\begin{tabular}{|c|c|c|c|c|c|c|}
\hline \multirow[b]{2}{*}{ Number of EOFs } & \multicolumn{2}{|c|}{ Mean } & \multicolumn{2}{|c|}{ Std dev } & \multicolumn{2}{|c|}{ Eddy forcing } \\
\hline & $200 \mathrm{hPa}$ & $500 \mathrm{hPa}$ & $200 \mathrm{hPa}$ & $500 \mathrm{hPa}$ & $200 \mathrm{hPa}$ & $500 \mathrm{hPa}$ \\
\hline 4 & -0.07 & -0.13 & 0.97 & 0.97 & 0.83 & 0.69 \\
\hline 6 & 0.04 & -0.05 & 0.99 & 0.99 & 0.93 & 0.86 \\
\hline 8 & 0.18 & 0.13 & 0.99 & 0.99 & 0.88 & 0.71 \\
\hline 10 & 0.42 & 0.43 & 0.98 & 0.98 & 0.84 & 0.74 \\
\hline
\end{tabular}


TABLE 6. Pattern correlation between various stochastic models and QG model for 200-hPa fields.

\begin{tabular}{lccc}
\hline & Mean & Std dev & Eddy forcing \\
\hline SMTV & 0.42 & 0.98 & 0.84 \\
MMTV & 0.57 & 0.98 & 0.74 \\
MRMTV-1 & 0.99 & 0.90 & 0.35 \\
MRMTV-2 & 0.99 & 0.95 & 0.42 \\
\hline
\end{tabular}

and multiplicative noises; these correction terms and noises account for the interaction of the resolved climate modes with the neglected nonclimate modes and the self-interaction of the nonclimate modes. The stochastic mode reduction strategy used here is suitable for implementation in complex geophysical systems with minimal fitting of the unresolved modes (Franzke a)

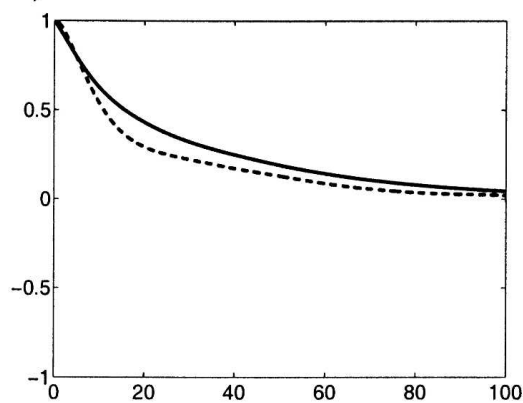

d)

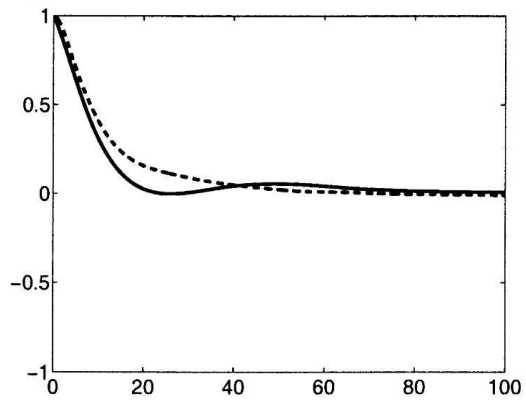

g)

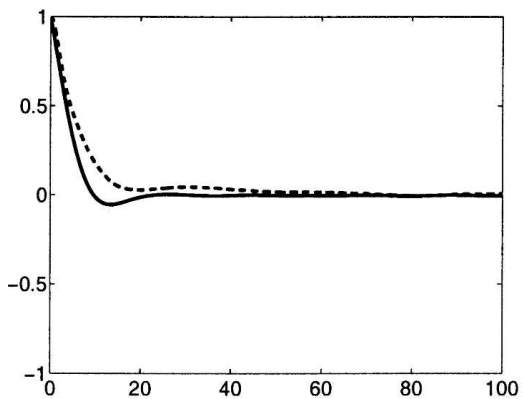

j)

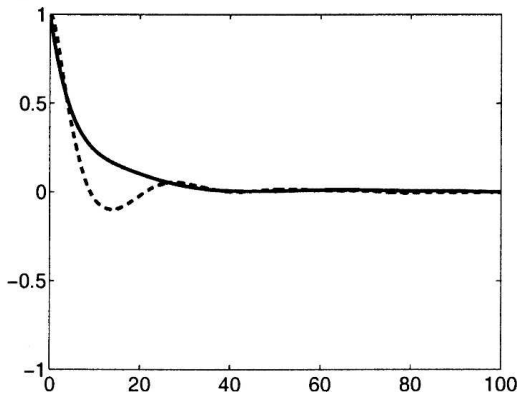

b)

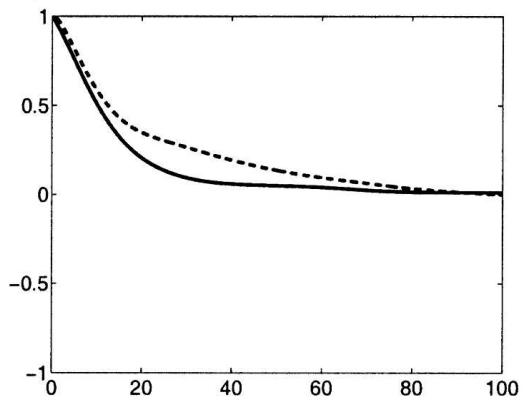

e)

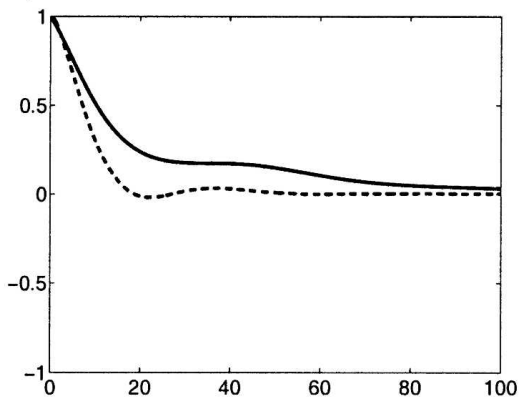

h)

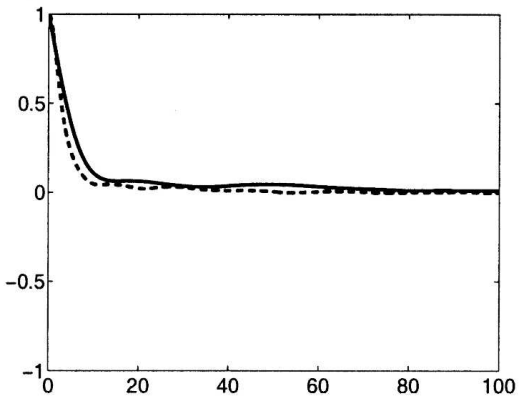

c)

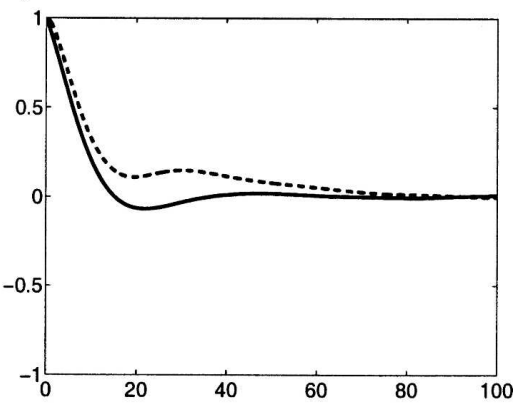

f)

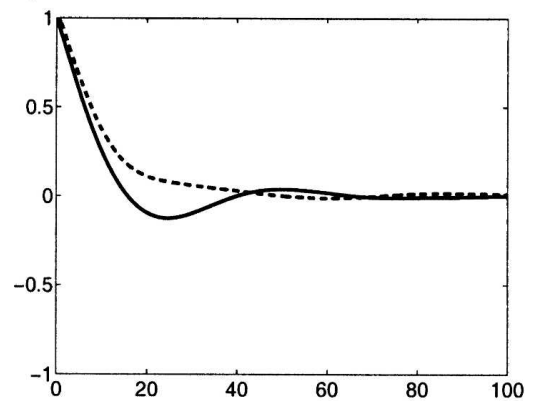

i)

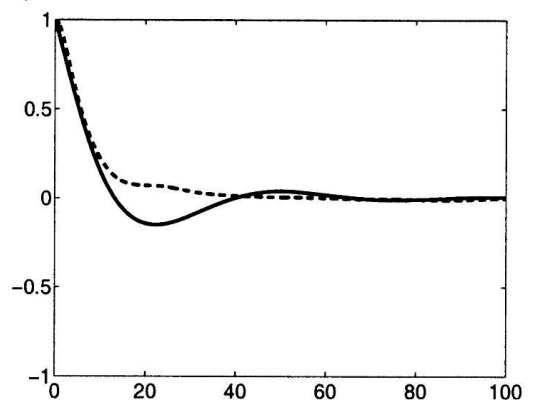

FIG. 11. Autocorrelations function of MMTV: (a) mode 1, (b) mode 2, (c) mode 3, (d) mode 4, (e) mode 5, (f) mode 6, (g) mode 7, (h) mode 8, (i) mode 9, and (j) mode 10 (solid line: stochastic model, dashed line: QG model). 
a)

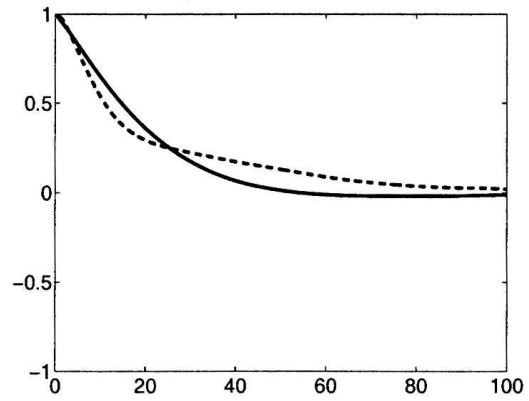

d)

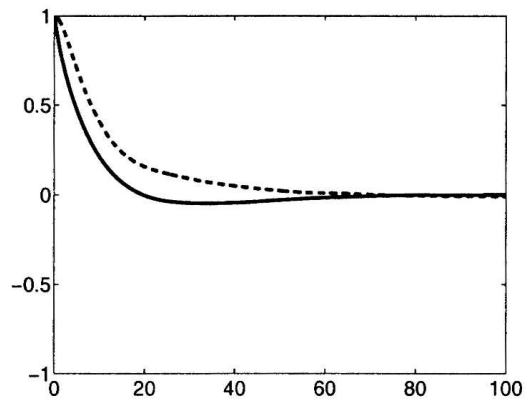

g)

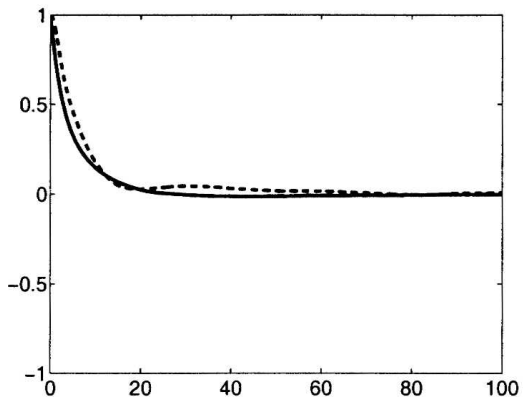

j)

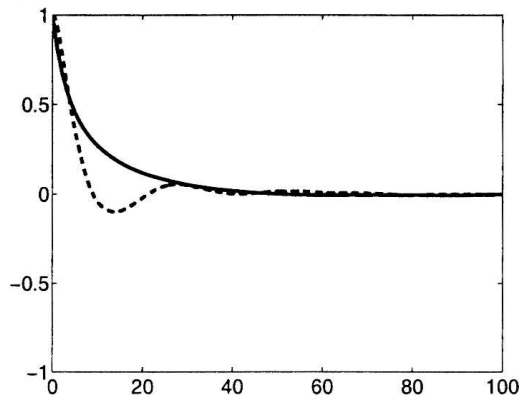

b)

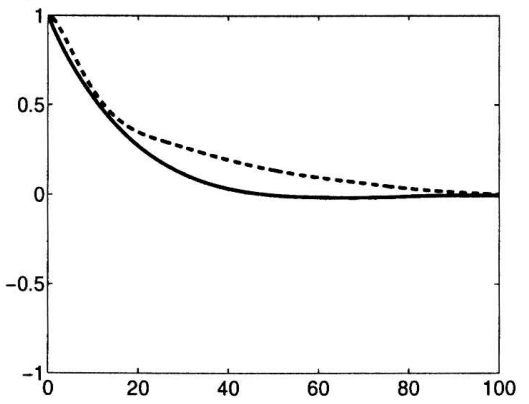

e)

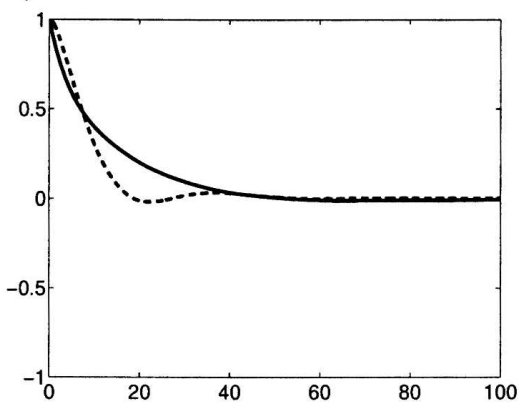

h)

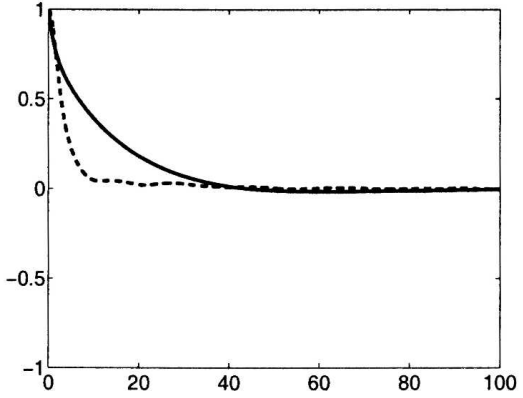

c)

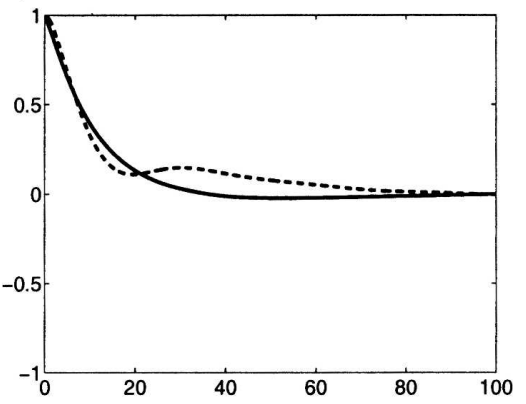

f)

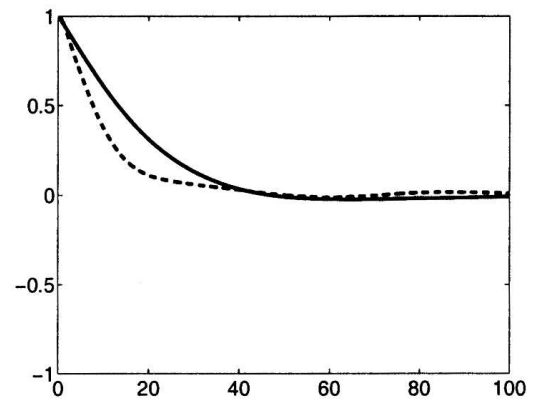

i)

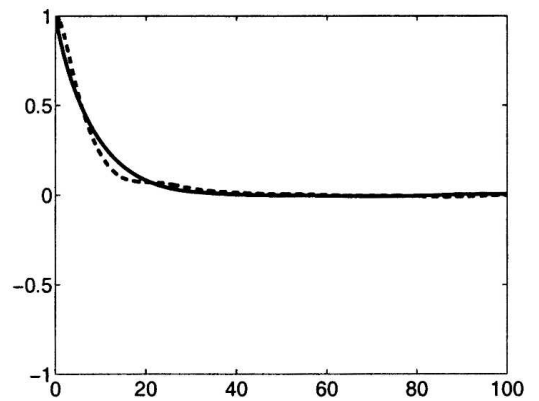

FIG. 12. Autocorrelation function of MRMTV-1: (a) mode 1, (b) mode 2, (c) mode 3, (d) mode 4, (e) mode 5, (f) mode 6, (g) mode 7, (h) mode 8, (i) mode 9, and (j) mode 10 (solid line: stochastic model, dashed line: QG model).

et al. 2005) and represents a generalized framework of that put forward originally in MTV99, MTV01, MTV02, and MTV03. In general, only an estimate for the variance and eddy turnover time is needed for each unresolved mode. To allow for systematic treatment of nonlinear interactions, the total energy metric is utilized rather than the geopotential height metric used typically for observations.
Low-order stochastic models with 10 or less resolved modes reproduce the geographical distributions of the standard deviation and eddy forcing well. They underestimate the standard deviations by at most a factor of about 1.5. Furthermore, they reproduce the autocorrelation functions reasonably well. A budget analysis shows that both linear and nonlinear correction terms as well as both additive and multiplicative noises are 
a)

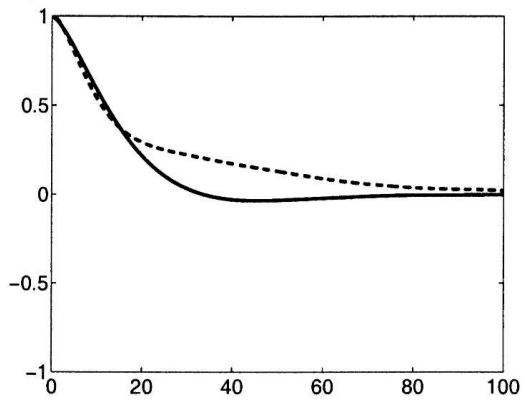

d)

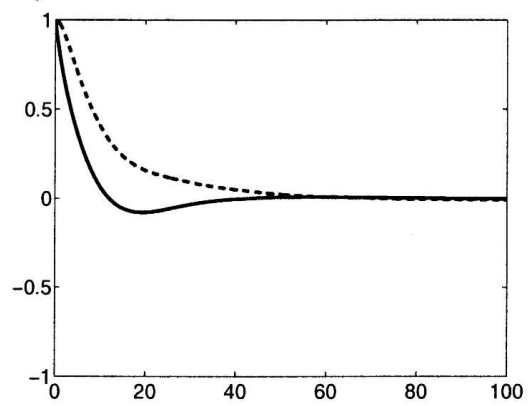

g)

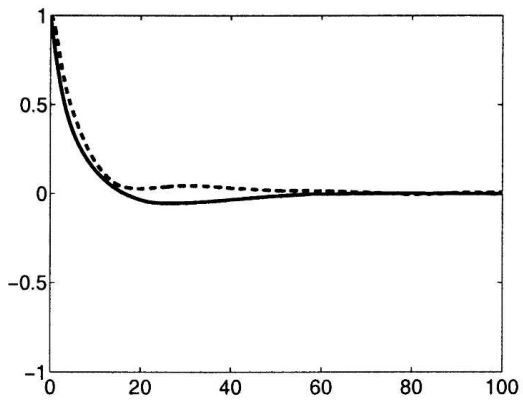

j)

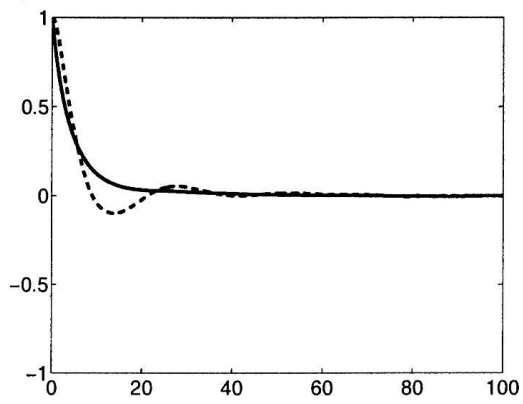

b)

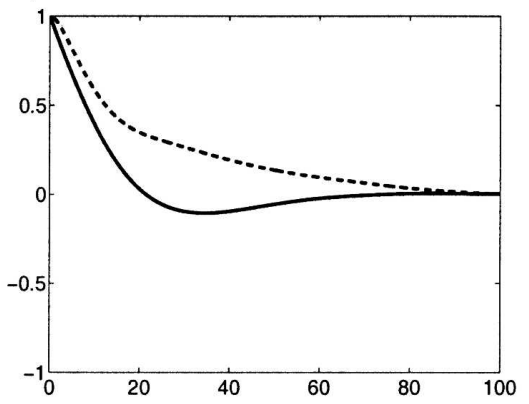

e)

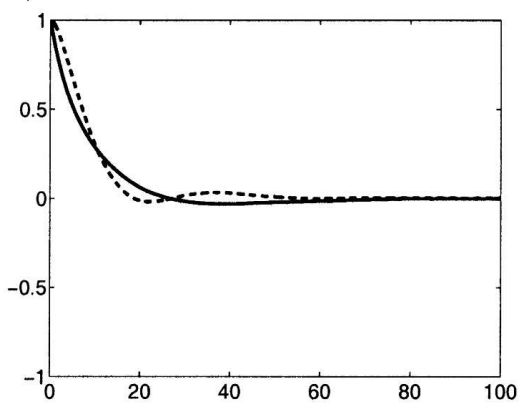

h)

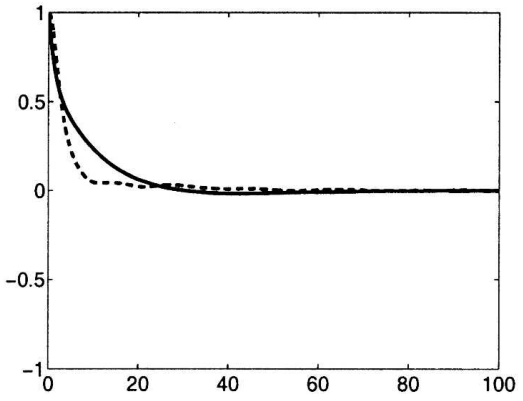

c)

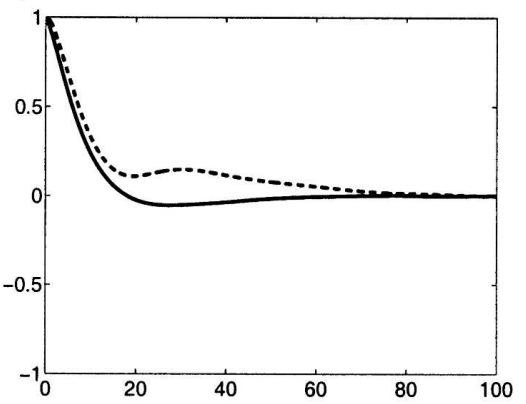

f)

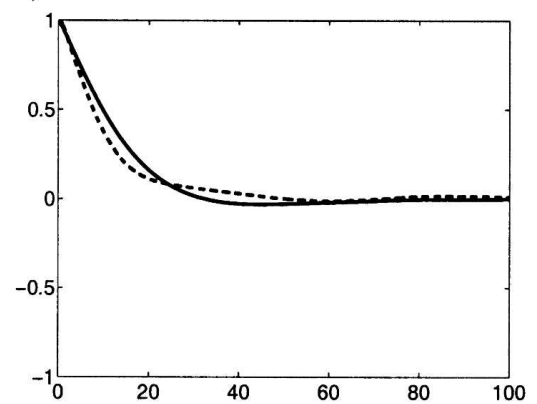

i)

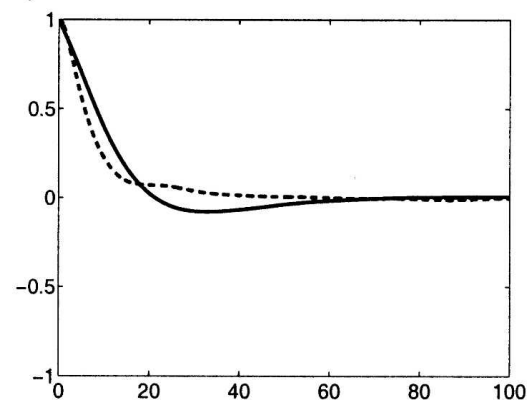

FIG. 13. Autocorrelation function of MRMTV-2: (a) mode 1, (b) mode 2, (c) mode 3, (d) mode 4, (e) mode 5, (f) mode 6, (g) mode 7, (h) mode 8, (i) mode 9, and (j) mode 10 (solid line: stochastic model, dashed line: QG model).

important. The physical intuition behind the noises is as follows: The additive noise stems from the linear interaction between the unresolved modes and the climatological mean state, and the multiplicative noise comes from the advection of the resolved modes by the unresolved modes. The functional form of these deterministic correction terms and noises (both additive and multiplicative) are predicted by the systematic stochastic mode reduction strategy, whereas, previous studies a priori approximate the nonlinear part of the equations by a linear operator and additive noise. This noise is typically white in time but may be spatially correlated. In other words, these studies truncate the dynamics on both the resolved and unresolved modes, and add white 
a)

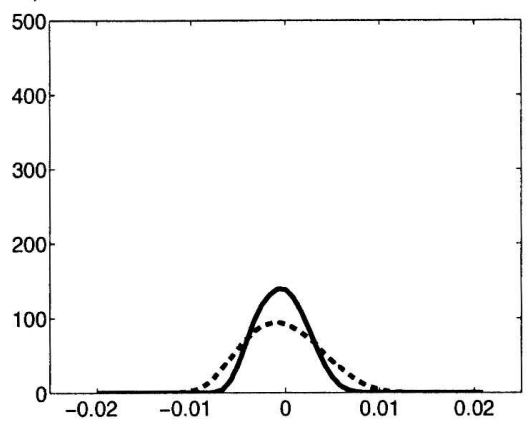

d)

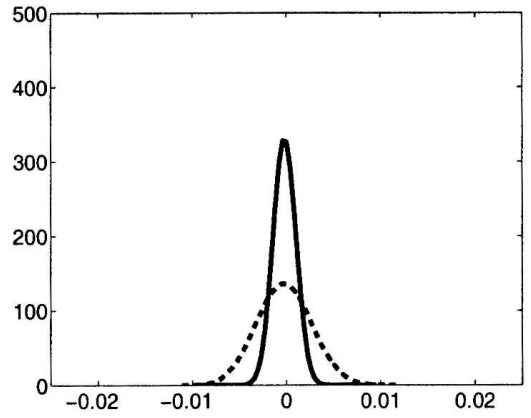

g)

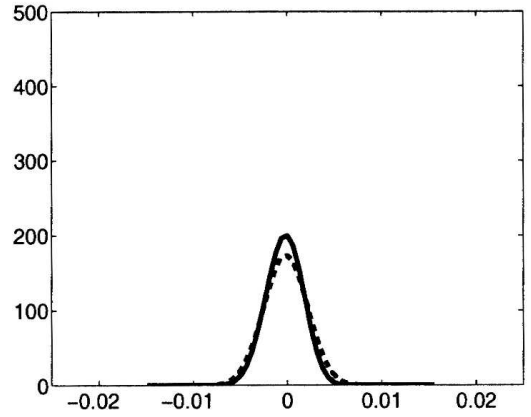

j)

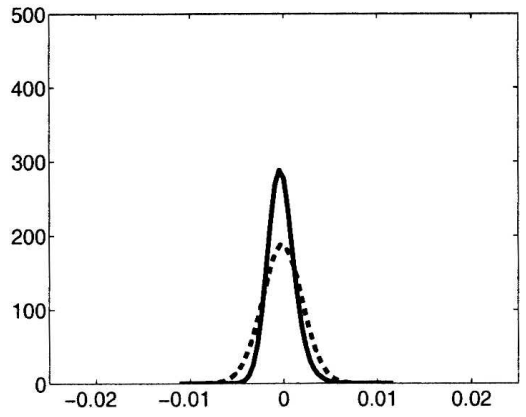

b)

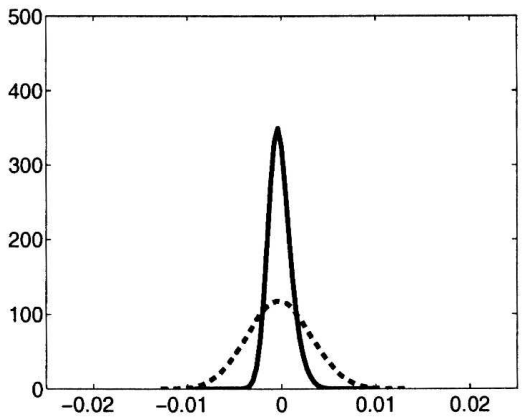

e)

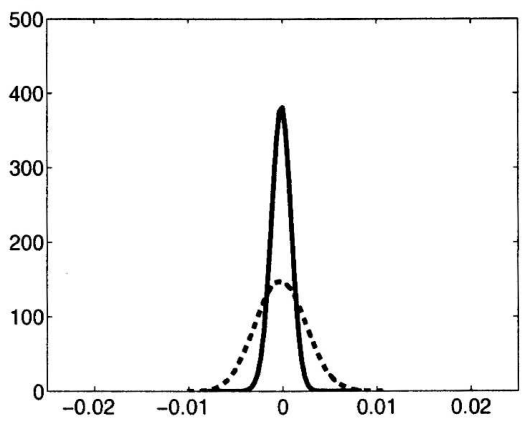

h)

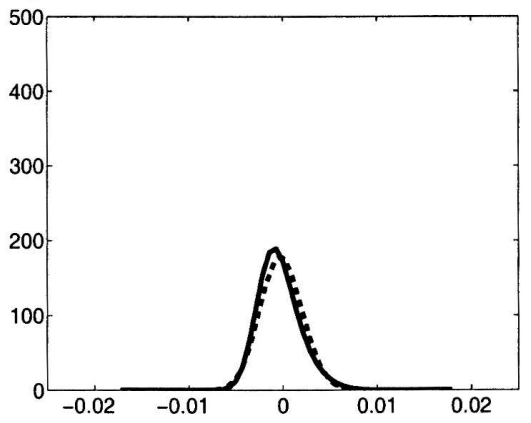

c)

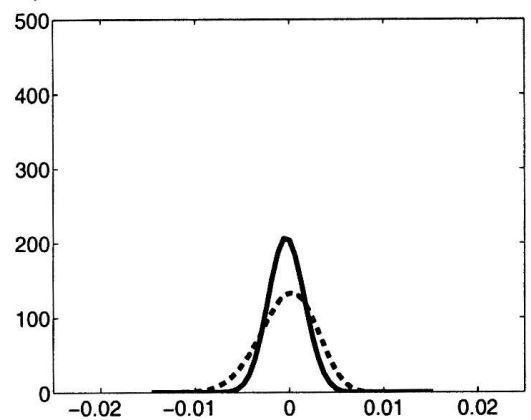

f)

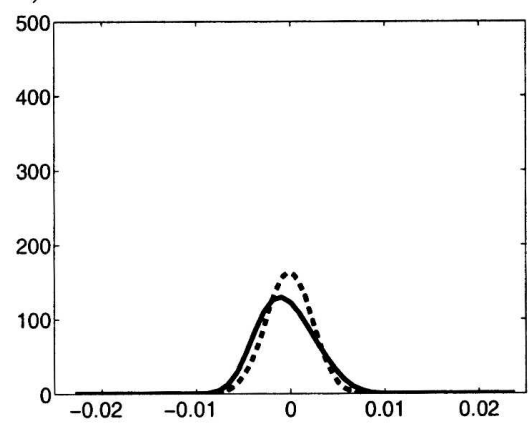

i)

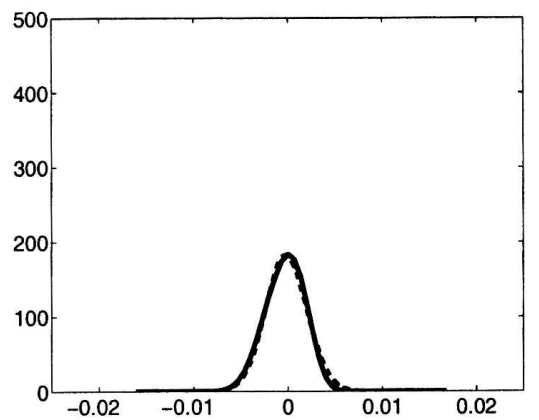

FIG. 14. PDF of MRMTV-2: (a) mode 1, (b) mode 2, (c) mode 3, (d) mode 4, (e) mode 5 , (f) mode 6, (g) mode 7, (h) mode 8, (i) mode 9, and (j) mode 10 (solid line: stochastic model, dashed line: QG model).

noise forcing together with damping in order to stabilize the linear model (Farrell and Ioannou 1993; Whitaker and Sardeshmukh 1998; Zhang and Held 1999). The systematic approach presented here truncates the dynamics only on the unresolved modes and only uti- lizes a stochastic ansatz for the nonlinear interactions among the unresolved modes themselves $\left(B^{\beta \beta \beta}\right)$ and predicts all necessary nonlinear correction terms and noises on the resolved modes; therefore, it also predicts the necessary damping on the resolved modes. 
The straightforward MTV stochastic climate models experience some climate drift. A minimal empirical MTV model without climate drift can be constructed by downscaling the bare truncation terms and upscaling the two important MTV processes (augmented linearity and multiplicative triads). As discussed in section 6, this allows the unrealistic climate drift from the bare truncation, reported in section 4 , to act on a longer time scale compared with the interactions of the unresolved modes. These empirical MTV stochastic climate models with minimal regression fitting still capture the geographical distribution of the standard deviation and eddy forcing and the autocorrelation functions reasonably well, while not experiencing climate drift. This surprising result can be interpreted as the fact that the resolved modes are predominantly driven by the unresolved modes and the self-interactions among the resolved modes are less important, as can already be seen from the bare truncation models, which do not capture any feature of the actual dynamics. Furthermore, these empirical MTV stochastic climate models suggest that the bare truncation is likely the cause of the climate drift. Integrations of bare truncation models (without any MTV correction terms) reported in section 4 already produce a big climate drift. The MTV mode reduction procedure is able to reduce the climate drift in most of the resolved modes, but is not able to overcome it completely. Previous results with a variety of simplified models show no climate drift in a MTV framework (MTV99; MTV01; MTV02; MTV03; Majda and Timofeyev 2004). This is likely because these simplified models are constructed in such a way that they already optimally capture the dynamics of the resolved modes. This gives further evidence that the total energy metric EOFs are not an adequate dynamical basis in capturing the dynamics of the slow modes.

Previous stochastic modeling studies utilized regression-fitted linear models with additive noise; these models a priori do not develop a climate drift, because these models are linearized around a basic state and the additive noise has zero mean. These studies tacitly model the constant forcing together with the nonlinear operator as linear damping plus white noise forcing and thus postulate no climate drift a priori (Farrell and Ioannou 1993; Newman et al. 1997; Whitaker and Sardeshmukh 1998; Zhang and Held 1999).

The results presented here suggest that the total energy metric EOFs do not capture adequately the slow dynamics of the system. Farrell and Ioannou (2001) also point to the potential inadequacy of EOFs as a basis for the dynamics. Their work refers to the study of the nonnormality of a stable linear operator and optimal representation of both the growing structures in the system and the structures into which these evolve. Kwasniok (1996, 2004) and Crommelin and Majda (2004) show that reduced models based on PIPs can be superior to low-order models based on EOFs. Recently, Kwasniok (2005, manuscript submitted to J. Atmos. $S c i$.) has introduced a simplified PIP strategy that only utilizes tendencies in the linear dynamics but allows for instantaneous linear instabilities. Obviously, it is worthwhile to explore the basic stochastic mode reduction strategy developed here in other optimal bases besides EOFs. The authors plan to do this in a future publication.

Acknowledgments. We thank Daan Crommelin, Eric Vanden-Eijnden, Frank Selten, Grant Branstator, and Ilya Timofeyev for beneficial discussions. Computing facilities were provided by the CGD group at NCAR. This research is funded through NSF-CMG Grant DMS-0222133 and ONR research Grant N00014-960043.

\section{APPENDIX A}

\section{Model Details}

Here we briefly summarize the dynamics of the QG model. A more detailed discussion can be found in Marshall and Molteni (1993). The model integrates prognostic equations for QG PV at 200 (level 1), 500 (level 2), and 800 (level 3) hPa of the form

$$
\begin{aligned}
& \frac{\partial q_{1}}{\partial t}=-J\left(\psi_{1}, q_{1}\right)-D_{1}\left(\psi_{1}, \psi_{2}\right)+S_{1} \\
& \frac{\partial q_{2}}{\partial t}=-J\left(\psi_{2}, q_{2}\right)-D_{2}\left(\psi_{1}, \psi_{2}, \psi_{3}\right)+S_{2} \\
& \frac{\partial q_{3}}{\partial t}=-J\left(\psi_{3}, q_{3}\right)-D_{3}\left(\psi_{2}, \psi_{3}\right)+S_{3},
\end{aligned}
$$

where the index $i=1,2,3$ refers to the pressure level. Here PV is defined as

$q_{1}=\nabla^{2} \psi_{1}-R_{1}^{-2}\left(\psi_{1}-\psi_{2}\right)+f$

$q_{2}=\nabla^{2} \psi_{2}+R_{1}^{-2}\left(\psi_{1}-\psi_{2}\right)-R_{2}^{-2}\left(\psi_{2}-\psi_{3}\right)+f$

$q_{3}=\nabla^{2} \psi_{3}+R_{2}^{-2}\left(\psi_{2}-\psi_{3}\right)+f\left(1+\frac{h}{H_{0}}\right)$,

where $f=2 \Omega \sin \phi, R_{1}=700 \mathrm{~km}$, and $R_{2}=450 \mathrm{~km}$ are Rossby radii of deformation, $h$ is the orographic height, and $H_{0}=9 \mathrm{~km}$ a scale height. The dissipative terms, indicated symbolically as $D_{1}, D_{2}$, and $D_{3}$, include contributions from temperature relaxation, Ekman dissipation, and horizontal diffusion 


$$
\begin{aligned}
D_{1}= & \frac{1}{\tau_{R}} R_{1}^{-2}\left(\psi_{1}-\psi_{2}\right)+\frac{1}{\tau_{H}} a^{8}[N(N+1)]^{-4} \nabla^{8} q_{1}^{\prime} \\
D_{2}= & \frac{1}{\tau_{R}} R_{1}^{-2}\left(\psi_{1}-\psi_{2}\right)-\frac{1}{\tau_{R}} R_{2}^{-2}\left(\psi_{2}-\psi_{3}\right) \\
& +\frac{1}{\tau_{H}} a^{8}[N(N+1)]^{-4} \nabla^{8} q_{2}^{\prime} \\
D_{3}= & -\frac{1}{\tau_{R}} R_{2}^{-2}\left(\psi_{2}-\psi_{3}\right)+\frac{1}{a \cos \phi}\left\{\frac{\partial}{\partial \lambda}\left[k(\lambda, \phi, h) v_{3}\right]\right. \\
& \left.-\frac{\partial}{\partial \phi}\left[k(\lambda, \phi, k) u_{3} \cos \phi\right]\right\} \\
& +\frac{1}{\tau_{H}} a^{8}[N(N+1)]^{-4} \nabla^{8} q_{3}^{\prime},
\end{aligned}
$$

and a planetary vorticity vector $\mathbf{f}$

where $\lambda$ is longitude, $\phi$ latitude, $h$ orographic height, $a$ the earth radius, and $u_{3}=-\left(\partial \psi_{3} / a \partial \phi\right), v_{3}=(1 / a$ $\cos \phi)\left(\partial \psi_{3} / \partial \lambda\right) ; q_{i}^{\prime}$ is the PV minus planetary vorticity and orographic component, and $N$ the total wavenumber. The drag coefficient $k$ is dependent on the landsea mask and orographic height

$$
k(\lambda, \phi, h)=\frac{1}{\tau_{E}}\left[1+c_{1} \operatorname{LS}(\lambda, \phi)+c_{2} \mathrm{FH}(h)\right],
$$

where $\tau_{E}=3$ days, $c_{1}=c_{2}=0.5 ; \operatorname{LS}(\lambda, \phi)$ is the fraction of land within a grid box; and

$$
\mathrm{FH}(h)=1-e^{-h / 1000 m} .
$$

Since LS and FH vary between 0 and $1, k$ varies between ( 3 days $)^{-1}$ over the oceans, $(2 \text { days })^{-1}$ over zeroaltitude land, and (1.5 days $)^{-1}$ over mountains higher than $2000 \mathrm{~m}$. The radiative time scale is $\tau_{R}=25$ days and the coefficient of the scale-selective diffusion is

$$
\mathbf{f}=\left[\begin{array}{c}
f \\
f \\
f\left(1+\frac{h}{H_{0}}\right)
\end{array}\right]
$$

the PV can be written in vector notation as

$$
\mathbf{q}=\nabla^{2} \boldsymbol{\psi}+\mathbf{E} \boldsymbol{\psi}+\mathbf{f}
$$

such that the spherical harmonics of total wavenumber $N$ are damped with a time scale $\tau_{H}=2$ days.

$$
\mathbf{E}=\left[\begin{array}{ccc}
-R_{1}^{-2} & R_{1}^{-2} & 0 \\
R_{1}^{-2} & -\left(R_{1}^{-2}+R_{2}^{-2}\right) & R_{2}^{-2} \\
0 & R_{2}^{-2} & -R_{1}^{-2}
\end{array}\right]
$$

and the streamfunction looks as follows:

$$
\boldsymbol{\psi}=\left(\boldsymbol{\nabla}^{2}+\mathbf{E}\right)^{-1}(\mathbf{q}-\mathbf{f})=\mathbf{Q}(\mathbf{q}-\mathbf{f}),
$$

with the understanding the $\mathbf{E}$ acts on all modes of a vertical level equally and $\mathbf{Q}=\left(\boldsymbol{\nabla}^{2}+\mathbf{E}\right)^{-1}$.

Equation (1) can now be written in form of a streamfunction equation as

$$
\frac{\partial \boldsymbol{\psi}}{\partial t}=\mathbf{Q}\left[-J\left(\boldsymbol{\psi}, \mathbf{Q}^{-1} \boldsymbol{\psi}+f+f \frac{h}{H}\right)-D(\boldsymbol{\psi})+S\right] .
$$

\section{APPENDIX B}

\section{Total Energy Metric and Interaction Coefficients}

The following total energy metric is defined (Ehrendorfer 2000):

$$
\mathbf{M}=\frac{\delta_{m}}{L}\left[\begin{array}{ccc}
n(n+1)+R_{1}^{-2} & -R_{1}^{-2} & 0 \\
-R_{1}^{-2} & n(n+1)+\left(R_{1}^{-2}+R_{2}^{-2}\right) & -R_{2}^{-2} \\
0 & -R_{2}^{-2} & n(n+1)+R_{2}^{-2}
\end{array}\right]
$$

where $\delta_{M}$ is defined to be $1 / 2$ for zonal mean modes, and to be equal to one for zonal wave modes, $n$ denotes the total wavenumber, and $L$ the number of vertical levels.

The streamfunction can now be expanded in total energy metric EOFs,

$$
\psi=\sum_{i} e_{i} p_{i}(t)+\bar{\psi}
$$

with the property that

$$
\left(e_{i}, e_{j}\right)=\int e_{i}^{T} \mathbf{M} e_{j} d \Omega=\int \tilde{e}_{i}^{T} \tilde{e}_{j} d \Omega=\delta_{i j},
$$

where $\tilde{e}_{i}=\mathbf{M}^{1 / 2} e_{i}$. By projecting the EOFs onto (3) we get the following equation set:

$$
\frac{\partial p_{i}(t)}{\partial t}=F_{i}+\sum_{j} L_{i j} p_{j}(t)+\sum_{j k} B_{i j k} p_{j}(t) p_{k}(t)
$$


with

$$
\begin{aligned}
F_{i}= & \int e_{i}^{T} \mathbf{M} \mathbf{Q} S d \Omega-\int e_{i}^{T} \mathbf{M} \mathbf{Q} D(\bar{\psi}) d \Omega \\
& -\int e_{i}^{T} \mathbf{M} \mathbf{Q} J(\bar{\psi}, f) d \Omega-\int e_{i}^{T} \mathbf{M} \mathbf{Q} J\left(\bar{\psi}, f \frac{h}{H}\right) d \Omega \\
& -\int e_{i}^{T} \mathbf{M} \mathbf{Q} J\left(\bar{\psi}, \mathbf{Q}^{-1} \bar{\psi}\right) d \Omega \\
L_{i j}= & -\int e_{i}^{T} \mathbf{M} \mathbf{Q} J\left(e_{j}, \mathbf{Q}^{-1} \bar{\psi}\right) p_{j}(t) d \Omega \\
& -\int e_{i}^{T} \mathbf{M} \mathbf{Q} J\left(\bar{\psi}, \mathbf{Q}^{-1} e_{j}\right) p_{j}(t) d \Omega \\
& -\int e_{i}^{T} \mathbf{M} \mathbf{Q} D\left(e_{j}\right) p_{j}(t) d \Omega \\
& -\int e_{i}^{T} \mathbf{M} \mathbf{Q} J\left(e_{j}, f\right) p_{j}(t) d \Omega \\
B_{i j k}= & -\int e_{i}^{T} \mathbf{M} \mathbf{Q} J\left(e_{j}, \mathbf{Q}^{-1} e_{k}\right) p_{j}(t) p_{k}(t) d \Omega . \\
& -\int e_{i}^{T} \mathbf{M Q} J\left(e_{j}, f \frac{h}{H}\right) p_{j}(t) d \Omega \\
&
\end{aligned}
$$

\section{APPENDIX C}

\section{Effective Equations}

For a detailed derivation of effective equations of the type as given in (5) see Franzke et al. (2005). Here we provide only the MTV coefficients.

The forcing coefficients are given by

$$
\tilde{H}_{i}=\tilde{H}_{i}^{(1)}+\tilde{H}_{i}^{(2)}
$$

with

$$
\begin{aligned}
& \tilde{H}_{i}^{(1)}=\sum_{j k l} \int_{-\infty}^{\infty} d t L_{j l}^{\alpha \beta} B_{i j k}^{\alpha \alpha \beta} \mathcal{B}_{l k}(t) \\
& \tilde{H}_{i}^{(2)}=\sum_{k l m n} \int_{-\infty}^{\infty} d t L_{k l}^{\beta \beta} B_{i m n}^{\alpha \beta \beta} \frac{\mathcal{B}_{k m}(t) \mathcal{B}_{l n}(t)+\mathcal{B}_{k n}(t) \mathcal{B}_{l m}(t)}{\mathcal{B}_{k}} .
\end{aligned}
$$

The new linear interaction coefficients are given by

$$
\tilde{L}_{j i}=\tilde{L}_{j i}^{(1)}+\tilde{L}_{j i}^{(2)}+\tilde{L}_{j i}^{(3)}
$$

with

$$
\begin{aligned}
\tilde{L}_{i j}^{(1)}= & \frac{1}{2} \sum_{k l} \int_{-\infty}^{\infty} d t L_{k j}^{\beta \alpha} L_{i l}^{\alpha \beta} \frac{\mathcal{B}_{k l}(t)}{\mathcal{B}_{k}} \\
\tilde{L}_{i j}^{(2)}= & \frac{1}{2} \sum_{k l m n} \int_{-\infty}^{\infty} d t B_{i m n}^{\alpha \beta \beta}\left(\frac{B_{l j k}^{\beta \alpha \beta}}{\mathcal{B}_{l}}+\frac{B_{k j l}^{\beta \alpha \beta}}{\mathcal{B}_{k}}\right)\left(\mathcal{B}_{k m}(t) \mathcal{B}_{l n}(t)\right. \\
& \left.+\mathcal{B}_{k n}(t) \mathcal{B}_{l m}(t)\right) \\
\tilde{L}_{i j}^{(3)}= & 2 \sum_{k l n} \int_{-\infty}^{\infty} d t B_{k j l}^{\alpha \alpha \beta} B_{i k n}^{\alpha \alpha \beta} \mathcal{B}_{l n}(t) .
\end{aligned}
$$

The new quadratic nonlinear interaction coefficients are given by

$$
\tilde{B}_{j i k}=\tilde{B}_{j i k}^{(1)}+\tilde{B}_{j i k}^{(2)}
$$

with

$$
\begin{aligned}
& \tilde{B}_{i j k}^{(1)}=\frac{1}{2} \sum_{m n} \int_{-\infty}^{\infty} d t B_{m j k}^{\beta \alpha \alpha} L_{i n}^{\alpha \beta} \frac{\mathcal{B}_{m n}(t)}{\mathcal{B}_{m}} \\
& \tilde{B}_{i j k}^{(2)}=\frac{1}{2} \sum_{m n} \int_{-\infty}^{\infty} d t\left(L_{m j}^{\beta \alpha} B_{i k n}^{\alpha \alpha \beta}+L_{m k}^{\beta \alpha} B_{i j n}^{\alpha \alpha \beta}\right) \frac{\mathcal{B}_{m n}(t)}{\mathcal{B}_{m}} .
\end{aligned}
$$

The new cubic nonlinear interaction coefficients are given by

$$
\begin{aligned}
\tilde{M}_{i j k l}= & \frac{1}{3} \sum_{m n} \int_{-\infty}^{\infty} d t\left(B_{n k l}^{\beta \alpha \alpha} B_{i j m}^{\alpha \alpha \beta}\right. \\
& \left.+B_{n l j}^{\beta \alpha \alpha} B_{i k m}^{\alpha \alpha \beta}+B_{n j k}^{\beta \alpha \alpha} B_{i l m}^{\alpha \alpha \beta}\right) \frac{\mathcal{B}_{n m}(t)}{\mathcal{B}_{n}} .
\end{aligned}
$$

The diffusion coefficients are given by

$$
Q_{i j}=Q_{i j}^{(1)}+Q_{i j}^{(2)}
$$

with

$$
\begin{aligned}
Q_{i j}^{(1)}= & \frac{1}{2} \sum_{l k} \int_{-\infty}^{\infty} d t L_{i l}^{\alpha \beta} L_{j k}^{\alpha \beta} \mathcal{B}_{l k}(t) \\
Q_{i j}^{(2)}= & \frac{1}{2} \sum_{k l m n} \int_{-\infty}^{\infty} d t B_{i k l}^{\alpha \beta \beta} B_{j m n}^{\alpha \beta \beta}\left(\mathcal{B}_{k m}(t) \mathcal{B}_{l n}(t)\right. \\
& \left.+\mathcal{B}_{k n}(t) \mathcal{B}_{l m}(t)\right)
\end{aligned}
$$

and

$$
U_{i j k}=\sum_{l n} \int_{-\infty}^{\infty} d t\left(L_{i l}^{\alpha \beta} B_{j k n}^{\alpha \alpha \beta}+L_{j l}^{\alpha \beta} B_{i k n}^{\alpha \alpha \beta}\right) \mathcal{B}_{l n}(t)
$$

$$
V_{j i k l}=2 \sum_{m n} \int_{-\infty}^{\infty} d t B_{i k m}^{\alpha \alpha \beta} B_{j l n}^{\alpha \alpha \beta} \mathcal{B}_{m n}(t) .
$$


Here $W^{(1)}=\left(W_{1}^{(1)}, \ldots, W_{m}^{(1)}\right)$ and $W^{(2)}=\left(W_{1}^{(2)}, \ldots\right.$, $\left.W_{m}^{(2)}\right)$ are two independent $m$-dimensional Wiener processes, and $\sigma_{j i}^{(1)}, \sigma_{j i}^{(2)}$ are defined so that

$$
\begin{gathered}
Q_{i j}^{(1)}+\sum_{k} U_{i j k} \alpha_{k}(t)+\sum_{k} \sum_{l} V_{i j k l} \alpha_{k}(t) \alpha_{l}(t) \\
=\sum_{k} \sigma_{i k}^{(1)}(\alpha(t)) \sigma_{j k}^{(1)}(\alpha(t)) \\
Q_{i j}^{(2)}=\sum_{k} \sigma_{i k}^{(2)} \sigma_{j k}^{(2)} .
\end{gathered}
$$

It is straightforward to verify that the left-hand sides in Eqs. (C17) and (C18) are positive definite $m \times m$ tensors, so the tensors $\sigma_{i j}^{(1)}[\alpha(t)]$ and $\sigma_{i j}^{(2)}$ exist and can be calculated by Cholesky decomposition.

The $\mathcal{B}_{i j}(s)$ are given by

$$
\mathcal{B}_{i j}(s)=\mathcal{B}_{j i}(-s)=\lim _{T-\infty} \frac{1}{T} \int_{0}^{T} d t \beta_{i}(t) \beta_{j}(t+s) .
$$

\section{REFERENCES}

Achatz, U., and G. W. Branstator, 1999: A two-layer model with empirical linear corrections and reduced order for studies of internal climate variability. J. Atmos. Sci., 56, 3140-3160.

—_, and J. D. Opsteegh, 2003a: Primitive-equation-based loworder models with seasonal cycle. Part I: Model construction. J. Atmos. Sci., 60, 466-477.

$\longrightarrow$, and 2003b: Primitive-equation-based low-order models with seasonal cycle. Part II: Application to complexity and nonlinearity of large-scale atmosphere dynamics. J. Atmos. Sci., 60, 478-490.

Berner, J., 2005: Linking nonlinearity and non-Gaussianity of planetary wave behavior by the Fokker-Planck equation. $J$. Atmos. Sci., 62, 2098-2117.

Blackmon, M. L., 1976: A climatological spectral study of the 500 $\mathrm{mb}$ geopotential height of the Northern Hemisphere. J. Atmos. Sci., 33, 1607-1623.

Branstator, G. W., 1990: Low-frequency patterns induced by stationary waves. J. Atmos. Sci., 47, 629-648.

—_, and S. E. Haupt, 1998: An empirical model of barotropic atmospheric dynamics and its response to tropical forcing. $J$. Climate, 11, 2645-2667.

— , and J. Frederiksen, 2003: The seasonal cycle of interannual variability and the dynamical imprint of the seasonally varying mean state. J. Atmos. Sci., 60, 1578-1592.

Crommelin, D. T., and A. J. Majda, 2004: Strategies for model reduction: Comparing different optimal bases. J. Atmos. Sci., 61, 2206-2217.

D'Andrea, F., and R. Vautard, 2001: Extratropical low-frequency variability as a low-dimensional problem. I: A simplified model. Quart. J. Roy. Meteor. Soc., 127, 1357-1374.

Delsole, T., 2001: Optimally persistent patterns in time-varying fields. J. Atmos. Sci., 58, 1341-1356.

_- 2004: Stochastic models of quasi-geostrophic turbulence. Surv. Geophys., 25, 107-149.

Ehrendorfer, M., 2000: The total energy norm in a quasigeostrophic model. J. Atmos. Sci., 57, 3443-3451.

Farrell, B. F., and P. J. Ioannou, 1993: Stochastic dynamics of baroclinic waves. J. Atmos. Sci., 50, 4044-4057. $\longrightarrow$, and - 2001: Accurate low-dimensional approximation of the linear dynamics of fluid flow. J. Atmos. Sci., 58, 27712789.

Feldstein, S. B., 2000: The time scale, power spectra, and climate noise properties of teleconnection patterns. J. Climate, 13, 4430-4440.

- 2002: The recent trend and variance increase of the annular mode. J. Climate, 15, 88-94.

Franzke, C., and S. B. Feldstein, 2005: The continuum and dynamics of Northern Hemisphere teleconnection patterns. $J$. Atmos. Sci., 62, 3250-3267.

— A. A. Majda, and E. Vanden-Eijnden, 2005: Low-order stochastic mode reduction for a realistic barotropic model climate. J. Atmos. Sci., 62, 1722-1745.

Gardiner, C. W., 1985: Handbook of Stochastic Methods Springer-Verlag, $442 \mathrm{pp}$.

Hasselmann, K., 1988: PIPs and POPs: The reduction of complex dynamical systems using principal interaction and oscillation patterns. J. Geophys. Res., 93, 11 015-11 021.

Hurrell, J. W., 1995: Decadal trends in the North Atlantic oscillation region temperatures and precipitation. Science, 269, 676-679.

Kondrashov, D., S. Kravtsov, and M. Ghil, 2006: Empirical mode reduction in a model of extratropical low-frequency variability. J. Atmos. Sci., in press.

Kravtsov, S., D. Kondrashov, and M. Ghil, 2005: Multilevel regression modeling of nonlinear processes: Derivation and applications to climate variability. J. Climate, 18, 4404-4424.

Kwasniok, F., 1996: The reduction of complex dynamical systems using principal interaction patterns. Physica D, 92, 28-60.

— 2004: Empirical low-order models of barotropic flow. J. Atmos. Sci., 61, 235-245.

Majda, A. J., and I. Timofeyev, 2000: Remarkable statistical behavior for truncated Burgers-Hopf dynamics. Proc. Natl. Acad. Sci. USA, 97, 12 413-12 417.

— intrinsic stochastic chaos: A paradigm model. Physica D, 199, 339-368.

—_ _ _ and E. Vanden-Eijnden, 1999: Models for stochastic climate prediction. Proc. Natl. Acad. Sci. USA, 96, $14687-$ 14691.

,-- , and 2001: A mathematical framework for stochastic climate models. Commun. Pure Appl. Math., 54, 891974.

$\longrightarrow,-$, and $\longrightarrow, 2002:$ A priori tests of a stochastic mode reduction strategy. Physica $D, \mathbf{1 7 0}, 206-252$.

,-- , and $\longrightarrow$ 2003: Systematic strategies for stochastic mode reduction in climate. J. Atmos. Sci., 60, 1705-1722.

$\longrightarrow$, R. Abramov, and M. Grote, 2005: Information Theory and Stochastics for Multiscale Nonlinear Systems. Monograph Series of Center for Research in Mathematics, American Mathematical Society, $140 \mathrm{pp}$.

Marshall, J., and F. Molteni, 1993: Toward a dynamical understanding of planetary-scale flow regimes. J. Atmos. Sci., 50, 1792-1818.

Newman, M., P. D. Sardeshmukh, and C. Penland, 1997: Stochastic forcing of the wintertime extratropical flow. J. Atmos. Sci., 54, 435-455.

Percival, D. B., J. E. Overland, and H. O. Mofjeld, 2001: Interpretation of North Pacific variability as a short- and longmemory process. J. Climate, 14, 4545-4559. 
Schubert, S. D., 1985: A statistical-dynamical study of empirically determined modes of atmospheric variability. J. Atmos. Sci., 42, 3-17.

Selten, F. M., 1993: Toward an optimal description of atmospheric flow. J. Atmos. Sci., 50, 861-877.

- 1995: An efficient description of the dynamics of barotropic flow. J. Atmos. Sci., 52, 915-936.

__, 1997: Baroclinic empirical orthogonal functions as basis functions in an atmospheric model. J. Atmos. Sci., 54, 20992114.

Stephenson, D. B., V. Pavan, and R. Bojariu, 2000: Is the North Atlantic Oscillation a random walk? Int. J. Climatol., 20, 1-18.

Sura, P., 2003: Stochastic analysis of Southern and Pacific Ocean sea surface winds. J. Atmos. Sci., 60, 654-666.

—- and J. Barsugli, 2002: A note on estimating drift and diffusion parameters from timeseries. Phys. Lett. A, 305, 304-311.
Thompson, D. W. J., and J. M. Wallace, 2001: Regional climate impacts of the Northern Hemisphere Annular mode and associated climate trends. Science, 293, 85-89.

Wallace, J. M., and D. S. Gutzler, 1981: Teleconnections in the geopotential height field during the Northern Hemisphere winter. Mon. Wea. Rev., 109, 784-804.

Whitaker, J. S., and P. D. Sardeshmukh, 1998: A linear theory of extratropical synoptic eddy statistics. J. Atmos. Sci., 55, 237 258

Winkler, C. R., M. Newman, and P. D. Sardeshmukh, 2001: A linear model of wintertime low-frequency variability. Part I: Formulation and forecast skill. J. Climate, 14, 4474-4494.

Zhang, Y., and I. M. Held, 1999: A linear stochastic model of a GCM's midlatitude storm tracks. J. Atmos. Sci., 56, 34163435. 